Février 2017

"Prédiction de l'usage des sols sur un zonage régulier à différentes résolutions et à partir de covariables facilement accessibles"

Raja Chakir, Thibault Laurent, Anne Ruiz-Gazen, Christine Thomas-Agnan, and Céline Vignes 


\title{
Prédiction de l'usage des sols sur un zonage régulier à différentes résolutions et à partir de covariables facilement accessibles
}

\author{
Raja Chakir ${ }^{*}$, Thibault Laurent ${ }^{* *}$, Anne Ruiz-Gazen ${ }^{* * *}$, Christine Thomas-Agnan $^{* * *}$, and \\ Céline Vignes ${ }^{* *}$ \\ *Economie Publique, INRA, AgroParisTech, Université Paris-Saclay, 78850 \\ Thiverval-Grignon, France \\ ${ }^{* *}$ Toulouse School of Economics, CNRS, University of Toulouse Capitole, 21 allée de \\ Brienne, 31042 Toulouse, France \\ ${ }^{* * *}$ Toulouse School of Economics, University of Toulouse Capitole, 21 allée de Brienne, \\ 31042 Toulouse, France
}

10 Janvier 2016

\section{Résumé}

Nous proposons dans ce travail d'évaluer dans quelle mesure un modèle de prédiction à partir de covariables facilement accessibles permet de donner une image de l'usage des sols comparable à celle obtenue à partir de l'information complète au niveau individuel souvent coûteuse. L'utilisation des sols est mesurée par l'occupation physique telle que définie dans l'enquête Teruti-Lucas, et nous considérons cinq catégories : urbain, agricole, forêts, prairies et sols naturels.

Notre approche comporte deux étapes : la première étape permet de modéliser l'utilisation du sol au niveau des points Teruti-Lucas tandis que la deuxième étape propose une méthode pour en déduire l'utilisation des sols sur un maillage défini par des carreaux. Le modèle de la première étape fournit des prédictions à un niveau fin. La deuxième étape agrège, en comparant plusieurs méthodes, ces prédictions sur les carreaux du maillage. Nous envisageons différents maillages réguliers du territoire en carreaux pour étudier la qualité de restitution en fonction de la résolution. Nous montrons qu'avec des variables facilement accessibles on obtient une qualité de prédiction acceptable au niveau point et que l'amélioration de la qualité est importante dès la première étape d'agrégation.

\section{Introduction}

Etant donnés les coûts élevés d'accès aux données individuelles, nous proposons dans ce travail d'évaluer dans quelle mesure un modèle de prédiction à partir de variables facilement accessibles permet de donner une image de l'usage des sols comparable à celle obtenue à partir de l'information complète au niveau individuel. Nous entendons par information complète celle provenant de l'enquête Teruti-Lucas 2010 du Ministère français de l'Agriculture et par image de l'usage des sols une prédiction de cet usage sur un maillage régulier du territoire en carreaux avec la possibilité de choisir le niveau de maillage. Les problèmes soulevés par cette étude sont tout d'abord le choix d'un modèle de prédiction au niveau de la résolution de la donnée de base, c'est-à-dire le point Teruti-Lucas, ensuite le choix d'une méthode pour agréger les prédictions ponctuelles au niveau des carreaux choisis (carreaux cibles) et enfin le choix d'un critère de qualité des résultats obtenus en fonction de la taille des carreaux cibles. 
Il existe un très grand nombre d'études sur la modélisation des usages des sols dans la littérature. Ces études peuvent être classées en différents groupes selon (1) les catégories de l'utilisation des sols examinées (rural vs urbain, agriculture vs forêt, et agriculture vs forêt vs urbain), (2) la résolution des données utilisées (données agrégées vs données individuelles), (3) la présence ou non de l'interaction spatiale, (4) la prise en compte ou non de la dimension dynamique et (5) la nature de la modélisation (statistique, économétrie, géographie, automate cellulaire) ${ }^{1}$.

L'objectif de la modélisation économétrique/statistique en général est soit de concevoir des modèles explicatifs ou des modèles prédictifs. La distinction entre ces deux types de modélisation n'est pas toujours facile et les contours des deux démarches ne sont pas souvent faciles à distinguer Shmueli, 2010. Pour résumer, l'objectif des modèles explicatifs est de tester des résultats théoriques qui peuvent être confirmés, infirmés ou précisés par l'estimation des paramètres. L'objectif des modèles prédictifs est de donner les prédictions les plus fiables. Ceci conduit à rechercher des modèles parcimonieux c'est-à-dire avec un nombre volontairement restreint de variables explicatives. Notre objectif dans cette étude est de nous concentrer essentiellement sur la qualité prédictive des modèles et non pas sur leur caractère explicatif.

Nous nous intéressons dans ce papier à la fois à la modélisation statistique et à la modélisation économétrique des usages des sols. Les modèles statistiques se placent en général dans une optique prédictive des usages des sols |Munroe et Müller, 2007; Lambin et al., 2000, Munroe et al., 2004; Veldkamp et Lambin, 2001, Verburg et al., 2004|. Les modèles économétriques peuvent être utilisés dans une optique prédictive ou explicative. Ces derniers cherchent à expliquer les usages des sols observés à l'aide de variables suggérées par la théorie économique ${ }^{2}$

Nous considérons une description de l'usage des sols en cinq catégories : urbain, agricole, forêts, prairies et sols naturels. Notre approche comporte deux étapes. La première étape permet de modéliser l'utilisation du sol au niveau des points Teruti-Lucas. La deuxième étape propose une méthode pour en déduire l'utilisation des sols sur un zonage défini par des carreaux. Dans la première étape, un modèle logit multinomial (MNL) d'utilisation du sol au niveau des points est estimé à partir des données de Teruti-Lucas grâce à des variables explicatives facilement accessibles telles que la base de Corine Land Cover (avec deux nomenclatures différentes), des variables météorologiques, des variables de qualité des sols, des variables socio-démographiques, l'altitude et le prix des terres. Une alternative utilisant des arbres de classement construits à partir des mêmes variables est ensuite comparée au modèle logit multinomial. La comparaison se fait en calculant des pourcentages de points bien prédits sur un sous-échantillon test, chacun des modèles ayant été ajusté sur un sous-échantillon d'apprentissage. Les résultats obtenus par les MNL et par les arbres sont très comparables.

Les modèles de la première étape permettent de fournir des prédictions à une résolution fine. Plus précisément, notre objectif est de comparer deux méthodes : la régression logistique multinomiale et l'arbre de classement. La régression logistique multinomiale est une méthode très classique dans l'étude de l'usage des sols sur données individuelles (voir McMillen 1989; Chomitz et Gray 1996; Nelson et Hellerstein 1997] et Chakir 2015 pour un survol) alors que l'arbre de classement est plus inédite dans ce contexte d'application. Pourtant cette méthode présente l'avantage d'être très simple et de permettre le choix automatique des variables. On considère ensuite un zonage du territoire en carreaux qui représente l'objectif de la restitution finale. La deuxième étape consiste à agréger, en comparant plusieurs méthodes, les prédictions issues de la première étape sur les carreaux du zonage. Nous proposons de mesurer la qualité d'estimation des probabilités au niveau

1. Voir Chakir 2015 pour une revue de la littérature sur les modèles économétriques d'usage des sols.

2. Voir Irwin et Geoghegan 2001; Irwin et Wrenn 2014 pour des revues de la littérature des modèles économétriques d'usage des sols et Chakir et Le Gallo 2013;; Chakir et Parent 2009] pour des exemples d'application dans le cas de la France. 
d'agrégation considéré par un score de Brier pondéré. Enfin, nous étudions la qualité de cette estimation en fonction du niveau de résolution considéré pour la restitution de l'image. Pour cela nous considérons plusieurs niveaux d'agrégation emboîtés et calculons les scores de Brier pondérés à chaque niveau. Une représentation graphique des gains en termes de score de Brier pondéré lors des agrégations successives est proposée comme une aide à la décision pour le choix du niveau de résolution minimal conduisant à une qualité d'estimation convenable. A notre connaissance, une telle comparaison entre différents niveaux d'agrégation est originale.

La section 2, portant sur les méthodes, présente la régression logistique multinomiale, les arbres de classement ainsi que la méthode du score de Brier pondéré. La section 3 décrit les données utilisées et le découpage en échantillon d'apprentissage et échantillon test. Nous y présentons également les différents découpages en carreaux considérés. Nous présentons dans la section 4 les résultats de l'ajustement des différents modèles ainsi que la comparaison de leur qualité de prédiction selon la méthode d'agrégation des probabilités estimées et selon le niveau d'agrégation spatiale.

\section{Méthodes}

\subsection{Régression logistique multinomiale (MNL)}

Afin d'estimer économétriquement les déterminants des usages des sols, le modèle théorique suggère que le propriétaire terrien maximise son utilité en comparant les bénéfices et les coûts de conversion des sols d'un usage à un autre à chaque date. Pour passer à la spécification économétrique, nous utilisons la cadre de l'approche de l'utilité aléatoire de McFadden [1974], qui permet de réécrire les revenus et les coûts de conversion de l'utilisation des sols comme des fonctions des variables observées et non observées. Ainsi l'utilité $U_{i k t}$ du propriétaire de la parcelle $i$ avec l'utilisation des sols $k$ à la date $t$ est la suivante :

$$
U_{i k t}=\beta x_{k i t}+\epsilon_{k i t} \quad \forall i=1, \ldots, n \quad, \forall k=1, \ldots, K \quad \text { et } \quad \forall t=1, \ldots, T
$$

où $x_{k i t}$ sont les variables explicatives observées, $\beta$ est le vecteur des paramètres à estimer et $\epsilon_{k i t}$ sont les termes d'erreur qui tiennent compte des variables non-observées qui pourraient influer sur l'utilité du propriétaire.

Nous supposons que le propriétaire a le choix entre $K$ catégories d'utilisation des sols pour chaque parcelle à chaque date. Le propriétaire foncier choisit l'utilisation optimale des sols de sa parcelle en comparant les utilités associées à chaque catégorie d'utilisation des sols. Si nous notons $y_{i t}=1,2, \ldots, K$ le choix d'utilisation des sols du propriétaire pour sa parcelle $i$ à la date $t$, nous avons :

$$
y_{i t}=k, \text { si } \quad U_{i k t} \geq \max _{j} U_{i j t} \quad \forall i=1, \ldots, n \quad, \forall k=1, \ldots, K \quad \text { et } \quad \forall t=1, \ldots, T
$$

Ainsi, la probabilité que la parcelle $i$ soit allouée à l'usage $k$ à la date $t$ s'écrit :

$$
P\left(y_{i t}=k\right)=\operatorname{Pr}\left[U_{i k t} \geq \max _{j} U_{i j t}\right], \quad \forall j=1, \ldots, K .
$$

McFadden 1974 identifie trois critères pour l'utilisation d'un modèle logit multinomial : l'indépendance, l'homoscédasticité et la distribution Gumbel des termes d'erreur. En supposant que ces conditions sont remplies, on peut montrer que la probabilité qu'une parcelle $i$ est en usage $k$ à la période $t$ est :

$$
p_{i k t}=\frac{\exp \left(\beta_{k} x_{k i t}\right)}{\sum_{k} \exp \left(\beta_{k} x_{k i t}\right)}
$$


Pour mesurer la qualité d'ajustement des modèles estimés, nous considérons plusieurs indicateurs classiques qui sont le critère d'information d'Akaike (AIC) [Akaike, 1974, le critère d'information bayésien (BIC) [Schwarz, 1978] et le $R^{2}$ de McFadden [McFadden, 1974] :

$$
\begin{gathered}
A I C=-2 L L+2 p \\
B I C=-2 L L+\log (n) \times p \\
R^{2} \text { de Mc Fadden }=1-\frac{L L}{L L_{0}}
\end{gathered}
$$

avec $L L$ la log-vraisemblance, $p$ le nombre de paramètres à estimer du modèle, $n$ la taille de l'échantillon et $L L_{0}$ la log-vraisemblance du modèle nul (sans autre paramètre que la constante). L'AIC et le BIC permettent de pénaliser les modèles en fonction du nombre de paramètres afin de satisfaire le critère de parcimonie, la pénalité étant encore plus grande avec le BIC. Plus ces critères sont faibles, meilleur est le modèle. Le $R^{2}$ de McFadden, ou pseudo- $R^{2}$, a été construit pour ressembler au $R^{2}$ de la régression linéaire mais doit s'interpréter en termes de part de déviance et non en termes de part de variance. Bien que compris entre 0 et 1 (comme le $R^{2}$ ), des simulations ont montré qu'une valeur autour de 0,3 correspond à une valeur élevée de $R^{2}$ (Domencich et McFadden 1975, p. 134-135]).

A l'issue de l'ajustement d'un modèle, nous obtenons des probabilités estimées par usage ainsi qu'éventuellement des prédictions d'usage en chaque point. Concernant les probabilités estimées au niveau des points, notons que les modèles MNL fournissent directement une valeur des probabilités estimées pour chaque usage. Si l'on souhaite aller plus loin en associant un usage prédit à chaque point, il faut un processus transformant les probabilités estimées en une prédiction d'usage. Pour les modèles MNL, il est classique de prédire l'usage correspondant à la probabilité estimée maximale. Notons que ce choix correspond au classifieur de Bayes qui minimise le risque de Bayes (Hastie et al. 2009, p. 21]). On pourrait également envisager de faire pour chaque point un tirage d'une loi multinomiale ayant pour paramètres les probabilités estimées des divers usages en ce point. Cette méthode s'avère mauvaise pour les prédictions au niveau des points mais nous reprendrons cette idée ensuite lorsque nous passerons à des niveaux plus agrégés pour la comparer à la prédiction basée sur la probabilité maximale. Ces prédictions étant faites, on peut alors calculer un taux de points bien classés qui est un critère de qualité de la prédiction, en affectant à chaque observation la catégorie d'usage de sol qui maximise la probabilité prédite et en divisant le nombre d'observations correctement prédites par le nombre total d'observations.

Pour vérifier si le pourcentage d'individus bien-classés est significativement meilleur que par un classement aléatoire, nous calculons la quantité suivante Hair, 2010] :

$$
Q_{P R E S S}=\frac{n(1-\tau \times K)^{2}}{K-1}
$$

où $n$ est la taille de l'échantillon test, $\tau$ est le taux de bien-classés dans l'échantillon test et $K$ le nombre de catégories. Si le classement est aléatoire, la statistique $Q_{P R E S S}$ suit une loi de $\chi^{2}$ à 1 degré de liberté.

La fonction mnlogit du package mnlogit [Zhiyu et Hasan, 2014] de R [Team, 2014] a été utilisée pour estimer les modèles de régression logistique multinomiale.

Nous avons estimés plusieurs modèles MNL, avec notamment un modèle économétrique (MNL-E) où le choix des variables est basé sur la théorie économique et un modèle statistique (MNL-Cm) où nous utilisons une procédure de sélection automatique. L'objectif de ce dernier modèle est 
d'arriver, à partir de l'ensemble des variables explicatives disponibles, à un modèle final qui retiendrait les variables qui s'avèrent significatives dans l'explication de la variation de la variable dépendante. Il existe plusieurs méthodes de sélection automatique de variables, parmi lesquelles la méthode pas-à-pas (en anglais « stepwise ») que nous avons retenue. Cette méthode, classique en régression [Jobson, 1999, consiste à examiner à chaque étape de la procédure à la fois si une nouvelle variable doit être ajoutée et si une des variables déjà incluse doit être éliminée. La procédure s'arrête lorsqu'aucune variable ne peut être rajoutée ou retirée du modèle selon le critère choisi (seuil de significativité, minimisation du BIC ou de l'AIC, etc.). Nous avons retenu comme critère la maximisation du taux de bien-classés dans l'échantillon test. Notons bien que ces méthodes de sélection automatique des variables ne se basent que sur des critères statistiques sans tenir compte du contexte économétrique.

\section{$2.2 \quad$ Arbres de classement}

Les arbres de classement constituent une alternative aux modèles de régression logistique multinomiale Tufféry, 2010]. Ce sont des arbres de décision ou de partitionnement où la variable à prédire est une variable qualitative à deux ou plusieurs modalités qui définissent des groupes. L'avantage de cette approche est qu'elle intègre une méthode automatique de choix des prédicteurs. L'idée est d'utiliser des prédicteurs qualitatifs ou quantitatifs pour diviser l'échantillon en nœuds ou segments successifs qui correspondent à des sous-échantillons les plus homogènes possible, au sens que les observations d'un nœud appartiennent majoritairement à un groupe. On considère des arbres binaires, c'est-à-dire tels que chacune des divisions conduit à deux nœuds. Le résultat est que l'ensemble des nœuds terminaux forme une partition de l'échantillon en classes homogènes. Chaque division est associée à un prédicteur déterminé par l'algorithme et la dichotomie s'effectue selon des valeurs prises par le prédicteur : sous-ensemble de modalités lorsque le prédicteur est qualitatif et seuil de division lorsque le prédicteur est quantitatif. Pour sélectionner la meilleure division possible, un indice d'homogénéité ou de pureté d'un nœud est choisi. Les étapes successives ainsi que la partition finale sont représentées graphiquement (voir la figure 4) sous la forme d'un arbre renversé, avec la racine en haut de l'arbre qui correspond à l'ensemble de l'échantillon, les divisions successives qui forment les branches et les nœuds terminaux qui constituent les feuilles. Pour obtenir une prédiction pour un individu de l'échantillon, on suit les branches de l'arbre qui correspondent aux valeurs des prédicteurs pour l'individu en question jusqu'au nœud terminal. Puis, par exemple, on adopte la règle majoritaire qui consiste à affecter l'observation à la classe la plus fréquente dans le nœud terminal. On obtient ainsi des règles de décision comme par exemple dans la figure 4 de nos résultats.

Il existe plusieurs algorithmes d'arbres de décision dont la méthode CART Breiman et al., 1984] qui consiste à construire une arbre maximal puis à l'élaguer. La fonction rpart du package rpart [Therneau et al., 2014] de R permet la mise en œuvre de cet algorithme pour les arbres de classement. La mesure de l'homogénéité d'un nœud prise par défaut dans rpart est l'indice de diversité de Gini que nous avons utilisé et qui permet une réduction de l'impureté des nœuds à chaque division. Parmi toutes les divisions binaires possibles, associées à tous les prédicteurs possibles, et pour chacun des nœuds, la meilleure division est donc choisie au sens de l'indice de Gini. Le critère d'arrêt de l'algorithme retenu par défaut par la fonction rpart est la taille minimale d'un nœud terminal qui doit être de 20 observations. Par ailleurs la vitesse de l'algorithme est améliorée en prenant en compte un paramètre dit de complexité, noté $c p$ et fixé par défaut à 0,01 . L'idée de ce paramètre est d'introduire un critère pénalisé par le nombre de segments terminaux et de ne pas diviser tous les nœuds, même s'ils contiennent suffisamment d'observations, lorsque le gain n'est pas suffisamment important au regard de la complexité grandissante de l'arbre. Dans notre cas, ce paramètre de complexité a été modifié à la valeur $c p=0,0001$ pour permettre la prise en compte d'un nombre suffisant de prédicteurs et la comparaison avec les MNL. 
Une fois l'arbre maximal obtenu, il est souvent conseillé de l'élaguer, c'est-à-dire de couper les branches les plus longues et les moins informatives, de façon à éviter que les dernières divisions soient instables et dépendent trop de l'échantillon d'apprentissage utilisé. L'algorithme CART utilisé pour l'élagage est composé de deux étapes. La première consiste à construire une suite de sous arbres de l'arbre maximal qui soient imbriqués et qui minimisent le paramètre de complexité. Le choix du sous arbre final dans la suite est ensuite obtenu par validation croisée. Cette procédure ne sera pas détaillée davantage ici mais est décrite en détail dans Breiman et al. [1984].

Alors que les modèles MNL nous fournissent des probabilités estimées, pour les arbres, il faut faire un calcul supplémentaire en estimant ces probabilités par la fréquence empirique correspondante au groupe de feuilles terminales associées à chaque usage. Par contre, la prédiction au point est directe car un usage est associé à chaque feuille terminale. A partir des prédictions, on peut calculer un taux de bien classés et comparer les résultats des arbres à ceux des MNL.

\subsection{Comparaison de la qualité de prédiction en fonction du niveau d'agréga- tion spatiale}

Nous utilisons le score de Brier Brier, 1950 pour juger de la qualité d'estimation des probabilités ou de prédiction de l'usage des sols. Ce score, initialement proposé dans le domaine de la météorologie, est une mesure d'erreur quadratique moyenne qui reste largement utilisée de nos jours au sein d'une plus large famille d'indicateurs de qualité Winkler et al., 1996, Buja et al. 2005 Merkle et Steyvers, 2013. Notre objectif est de déterminer des niveaux d'agrégation qui conduisent à une bonne qualité de prédiction et de mettre en garde l'utilisateur contre l'usage de niveaux trop fins pour assurer une qualité de prédiction ou d'estimation acceptable.

Le score de Brier est défini par :

$$
\text { Score de Brier }=\frac{1}{2 n} \sum_{k=1}^{K} \sum_{i=1}^{n}\left(z_{i k}-\hat{p}_{i k}\right)^{2}
$$

avec

$$
z_{i k}= \begin{cases}1 & \text { si l'usage } k \text { est observé au point } i \\ 0 & \text { sinon }\end{cases}
$$

et $\hat{p}_{i k}$ est la probabilité estimée d'observer l'usage $k$ au point $i$ pour $k=1, \ldots, K$ et $i \in\{1, \ldots, n\}$.

Le score de Brier varie entre 0 et 1 . Plus sa valeur est faible, meilleure est la qualité. On peut calculer ce score à différents niveaux d'agrégation. Dans notre application, le niveau le plus désagrégé est le niveau ponctuel (point Teruti-Lucas) tandis que le niveau le plus agrégé est la région Midi-Pyrénées. Ainsi, pour un vecteur de probabilités estimées obtenu par le modèle multinomial logit MNL-Cm tel que détaillé en section 4, on observe un score de Brier au niveau ponctuel (appelé score individuel) de 0,2502 tandis que le score au niveau de la région (appelé score global) est de $3,49 \times 10^{-6}$. Intuitivement, on s'attend à une décroissance du score de Brier et donc à une amélioration de la qualité de prédiction au fur et à mesure que l'on agrège les données. On peut montrer toutefois que cette propriété n'est pas vérifiée par le score de Brier car celui-ci ne prend pas en compte la taille des groupes agrégés. Nous proposons une mesure adaptée du score de Brier, appelée score de Brier pondéré et notée B, qui permet de pallier à cet inconvénient.

Le score de Brier pondéré est défini pour des groupes $G_{g}\left(g \in I_{G}\right)$ formant une partition de 
$\{1, \ldots, n\}$ par :

$$
\mathrm{B}_{G}=\frac{1}{2 n} \sum_{k=1}^{K} \sum_{g \in I_{G}} \# G_{g}\left(\bar{z}_{g k}-\overline{\hat{p}}_{g k}\right)^{2}
$$

avec

$$
\begin{aligned}
& \bar{z}_{g k}=\frac{1}{\# G_{g}} \sum_{i \in G_{g}} z_{i k} \text { est la fréquence observée de l'usage } k \text { dans le groupe } G_{g} \\
& \overline{\hat{p}}_{g k}=\frac{1}{\# G_{g}} \sum_{i \in G_{g}} \hat{p}_{i k} \text { est la probabilité estimée de l'usage } k \text { dans le groupe } G_{g}
\end{aligned}
$$

Dans notre application, les groupes $G_{g}$ seront par exemple les segments Teruti-Lucas ou des agrégations de segments.

Notons que le niveau individuel est nécessaire pour calculer la taille des groupes $G_{g}$ dans la définition du score pondéré alors que seul le nombre de groupes est nécessaire au calcul du score de Brier.

Par ailleurs, le score de Brier pondéré global est équivalent au score de Brier global :

$$
\mathrm{B}_{\text {global }}=\frac{1}{2} \sum_{k=1}^{K}\left(\bar{z}_{k}-\overline{\hat{p}}_{k}\right)^{2}
$$

avec

$$
\begin{aligned}
& \bar{z}_{k}=\frac{1}{n} \sum_{i=1}^{n} z_{i k} \text { est la fréquence observée de l'usage } k \text { sur l'ensemble de l'échantillon } \\
& \overline{\hat{p}}_{k}=\frac{1}{n} \sum_{i=1}^{n} \hat{p}_{i k} \text { est la probabilité estimée de l'usage } k \text { sur l'ensemble de l'échantillon }
\end{aligned}
$$

Ce score global est nul dans le cas de la régression logistique multinomiale si des constantes spécifiques aux alternatives sont introduites dans le modèle. En effet la probabilité estimée pour chaque alternative est égale à la fréquence observée de cette alternative dans l'échantillon (Train 2009 , p. 62]).

La propriété suivante montre que le score de Brier pondéré agrégé à un niveau donné $\left(G_{g}\right)$ s'écrit comme la somme du score pondéré agrégé à un niveau moins fin $\left(J_{j}\right)$ et de la variance intra des erreurs de prédiction dans les groupes les moins fins (à un facteur multiplicatif près). La preuve de ce résultat est donnée en annexe 6.3. On en conclut que le score de Brier pondéré décroît lorsque l'on agrège des zones et que l'on moyenne les probabilités estimées sur ces zones. Cette décroissance est d'autant plus marquée que la variabilité des erreurs de prédiction est importante dans les zones qui vont être agrégées.

$$
\mathrm{B}_{G}=\mathrm{B}_{J}+\frac{1}{2 n} \sum_{k=1}^{K} \sum_{j \in I_{J}} \sum_{g \in I_{G_{j}}} \# G_{g}\left(\bar{z}_{g k}-\overline{\hat{p}}_{g k}-\bar{z}_{j k}+\overline{\hat{p}}_{j k}\right)^{2}
$$

où les groupes $J_{j}\left(j \in I_{J}\right)$ forment une partition de $\{1, \ldots, n\}$ plus agrégée que les groupes $G_{g}$ telle que les $I_{G_{j}}\left(j \in I_{J}\right)$ forment une partition de $I_{G}$ et $J_{j}=\cup_{g \in I_{G_{j}}} G_{g}$ avec $j \in I_{J} ; \bar{z}_{j k}=\frac{1}{\# J_{j}} \sum_{i \in J_{j}} z_{i k}$ et $\overline{\hat{p}}_{j k}=\frac{1}{\# J_{j}} \sum_{i \in J_{j}} \hat{p}_{i k}$. 
Ainsi, plus le niveau d'agrégation est fin, plus le score de Brier pondéré est élevé et pire est la prédiction ou l'estimation.

Pour choisir parmi plusieurs niveaux d'agrégation $A_{1}, \ldots, A_{L}$ où $A_{1}$ (resp. $A_{L}$ ) désigne le niveau le plus (resp. le moins) fin, nous proposons de comparer graphiquement les différences entre scores de Brier pondérés successifs $B_{l-1}-B_{l}$ divisées par le score de Brier individuel noté $B_{0}$. Ces ratios successifs mesurent le gain en termes de qualité lorsque l'on passe du niveau d'agrégation $A_{l-1}$ à $A_{l}$. Leur somme de $l=1$ à $L$ vaut 1 . La section 4.2 illustre la mise en ouvre de cette méthode et l'interprétation du graphique pour le choix du niveau d'agrégation dans le cadre de l'enquête Teruti-Lucas.

\section{Données}

Notre zone d'étude est la région Midi-Pyrénées qui est la plus vaste région française (45 $348 \mathrm{~km}^{2}$, soit $8,3 \%$ du territoire national) et celle comportant le plus grand nombre de communes (3020 communes au 1er janvier 2013). C'est une région plutôt rurale (cf. figure 1) qui n'abrite que 4,5\% de la population métropolitaine (2 903420 habitants en 2011 3 . Elle présente l'avantage d'une certaine diversité d'usage des sols avec Toulouse, le grand pôle urbain, et de vastes zones agricoles au centre, les Pyrénées au sud et des forêts et des prairies au nord (cf. figure 3 ).

Figure 1 - Midi-Pyrénées : Zonage en aires urbaines

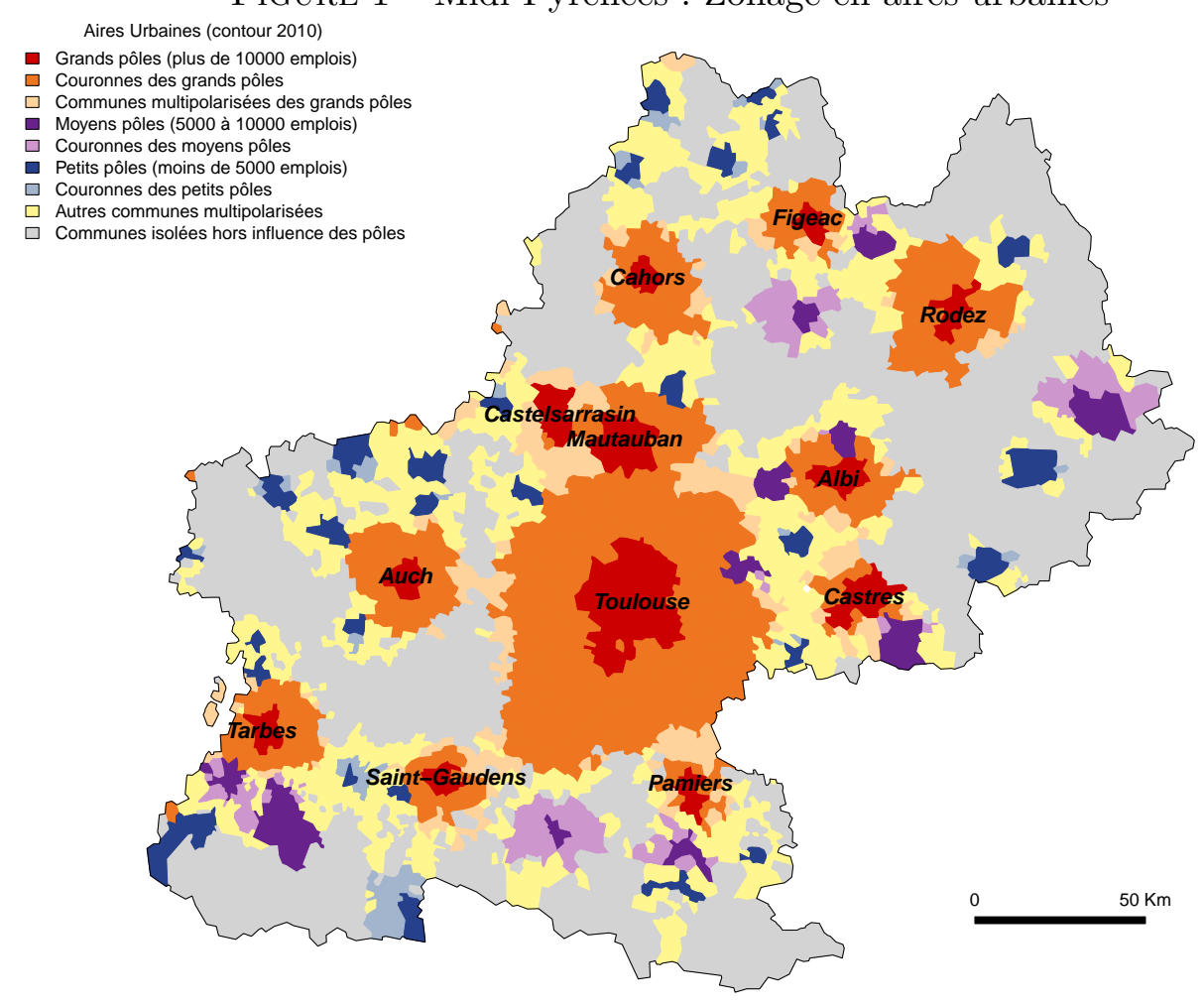

\subsection{Variable d'intérêt Usage $d u$ sol : données Teruti-Lucas 2010}

Les données d'usage des sols proviennent de l'enquête Teruti-Lucas, réalisée par le Service de la Statistique et de la Perspective (SSP) du Ministère de l'Agriculture. Cette enquête permet de

3. source : Insee, http://www.insee.fr/fr/regions/midi-pyrenees/default.asp?page=faitsetchiffres/ presentation/essentiel_mp.htm 
renseigner sur l'évolution de l'occupation des sols au niveau d'un échantillon de points répartis sur l'ensemble du territoire français. L'enquête repose sur l'association de photographies aériennes et d'enquêtes de terrain. Cette enquête a débuté en 1982 mais l'échantillon de points a été entièrement renouvelé en 1991 et 1992 et la nomenclature des usages des sols a été modifiée. En 2005, l'enquête Teruti a évolué et l'échantillon de points a été totalement renouvelé pour (i) améliorer la précision de l'enquête en utilisant les progrès réalisés en matière de géo-référencement et de traitement de données cartographiques et (ii) permettre une cohérence de nomenclature et de méthode avec une enquête similaire réalisée à l'échelle européenne : l'enquête européenne LUCAS (Land Use/Cover Area frame statistical Survey). La nouvelle enquête s'appelle Teruti-Lucas.

Dans l'enquête Teruti-Lucas, chaque segment comporte 25 points alignés par 5 (cf. figure 2 ) et seuls les 10 points des deux premières lignes sont disponibles dans les données que nous utilisons ensuite (les autres ont été créés pour d'éventuelles extensions de l'enquête). L'échantillon pour la période 2006-2010 est donc constitué de 309080 points pour la France métropolitaine et de $n=25317$ points pour la région Midi-Pyrénées, observés chaque année.

Figure 2 - Enquête Teruti-Lucas : segments et points

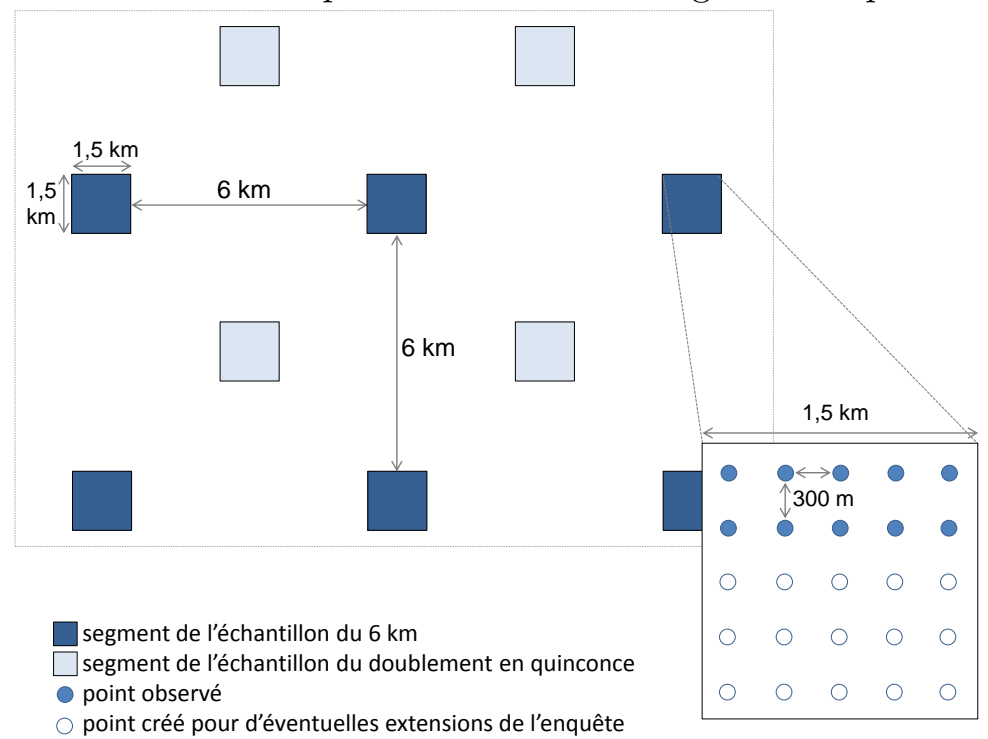

On s'intéresse uniquement à l'occupation physique des sols, et non à l'utilisation fonctionnelle qui est l'autre principale variable recueillie et qui mesure la destination socio-économique du territoire observé. L'occupation physique des sols est directement déduite de l'observation. Elle est codée selon deux nomenclatures : une nomenclature de synthèse en 57 postes, qui permet la continuité avec la version précédente de l'enquête en France (Teruti 1992-2004) et une nomenclature en 122 postes, complètement remaniée mais qui recouvre les mêmes catégories d'usage. Nous utiliserons la nomenclature en 57 postes, regroupés en cinq catégories : usage urbain, usage agricole, forêts, prairies et sols naturels. Ces cinq catégories sont pertinentes pour étudier par exemple l'impact des changements d'usage des sols sur les émissions de gaz à effets de serre et plus exactement sur la séquestration de carbone. De plus, étant données les variables explicatives dont on dispose, il serait difficile de détailler l'usage agricole par exemple. En effet l'allocation des sols entre blé, orge et colza fait partie des décisions basées sur des considérations agronomiques (rotations, système de production, ...) qu'on n'observe pas dans nos données. La figure 3 illustre l'usage majoritaire au segment et l'annexe 6.1 fournit la correspondance entre la nomenclature en 57 postes et notre variable d'intérêt, le regroupement en cinq catégories. 
Figure 3 - Usage des sols majoritaire dans le segment

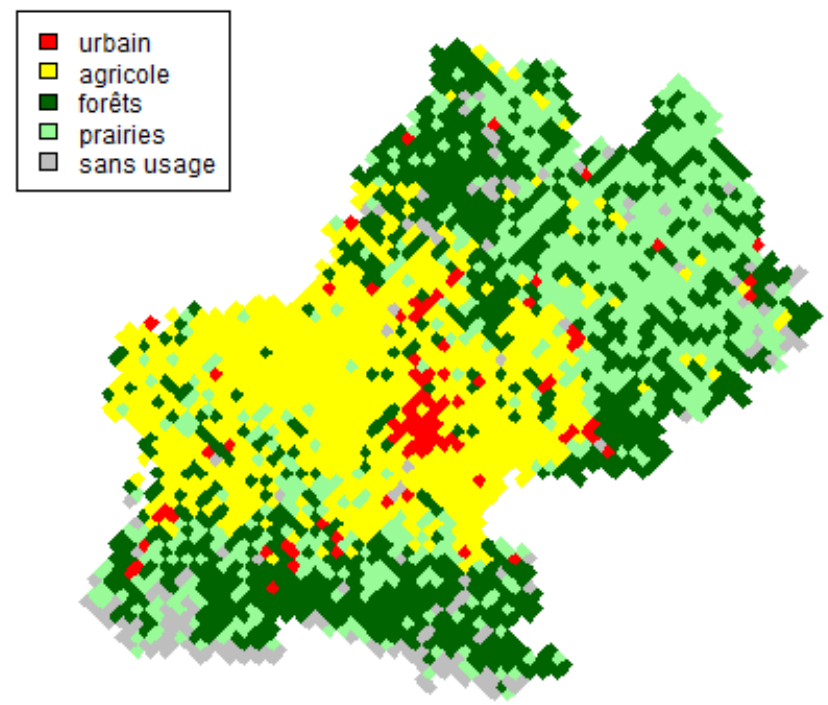

\subsection{Variables explicatives}

Les variables explicatives retenues proviennent de multiples bases de données disponibles à des niveaux géographiques différents. Le tableau 1 dresse une synthèse de ces bases de données et des variables en découlant. L'annexe 6.2 fournit des compléments d'information sur ces bases, notamment un résumé des aires des différentes zones (CLC, UCS, NRA, communes) dans le tableau 9 et les statistiques descriptives des variables retenues dans les modèles (tableaux 10 à 15). Les variables mesurées avec la résolution la plus fine sont l'occupation du sol au sens de Corine Land Cover et l'altitude.

Utilisant des outils d'économétrie spatiale, nous avons aussi introduit des variables spatialement décalées parmi les variables explicatives. En effet, l'occupation des sols (CLC), la densité de population, les parts d'agriculteurs et de cadres ainsi que l'altitude ont été mesurés aux points voisins. Nous considérons que les voisins d'un point Teruti-Lucas sont les points appartenant au même segment. Pour les identifier et construire les variables spatialement décalées, nous avons défini une matrice de voisinage basée sur un seuil de distance de $1400 \mathrm{~m}$. La matrice est normalisée par une standardisation en ligne. Ainsi, les variables quantitatives spatialement décalées représentent la valeur moyenne des autres points du segment. L'occupation des sols étant une variable qualitative, nous avons plutôt choisi de retenir la valeur majoritaire dans le segment et de ne pas utiliser la matrice de voisinage. Les données manquantes pour les variables spatialement décalées (c'est-à-dire point Teruti-Lucas seul dans le segment) sont exclues des analyses.

\subsection{Echantillon d'apprentissage et échantillon test}

Afin de pouvoir évaluer le pouvoir prédictif des modèles et la validité externe des résultats obtenus, nous utilisons la stratégie consistant à diviser l'échantillon d'origine en deux parties, l'une dite échantillon d'apprentissage servant à construire un modèle et l'autre dite échantillon test servant à comparer la réalité observée à sa prédiction par ce modèle. Pour cela nous divisons l'ensemble des segments Teruti-Lucas en deux parties : l'échantillon dit « du doublement en quinconce » comportant 12657 points servant d'échantillon d'apprentissage (en clair dans la figure 2) et l'échantillon test dit « du $6 \mathrm{~km}$ doublé » comportant 12660 points ${ }^{4}$. De ce fait la répartition des points de chaque échantillon sur le territoire est bien régulière.

3. Les statistiques descriptives de l'aire de ces zones sont données dans le tableau 9 de l'annexe 6.2

4. voir http://agreste.agriculture.gouv.fr/IMG/pdf/teruti2014methobsva.pdf 
TABleau 1 - Sources des données

\begin{tabular}{|c|c|c|c|c|}
\hline nom & niveau géographique & source & année & unité \\
\hline usage des sols & points espacés de $6 \mathrm{~km}$ & Teruti-Lucas & 2010 & - \\
\hline occupation des sols (CLC) & zones $(>25 \mathrm{ha})^{3}$ & Corine Land Cover & 2006 & - \\
\hline altitude & points espacés de $250 \mathrm{~m}$ & BDAlti de l'IGN & - & mètres \\
\hline nature du sol & zones $\mathrm{UCS}^{3}$ & $\begin{array}{l}\text { BGSF (@ INRA, Unité } \\
\text { INFOSOL, Orléans) }\end{array}$ & 1998 & - \\
\hline $\begin{array}{l}\text { texture dominante en sur } \\
\text { matériau de base } \\
\text { évolution de la texture du } \\
\text { présence d'une couche im }\end{array}$ & sol & & & $\begin{array}{l}- \\
- \\
-\end{array}$ \\
\hline météorologie & grille $25 \times 25 \mathrm{~km}$ & $\begin{array}{l}\text { JRC-MARS Agri4cast } \\
\text { Meteorological Data Base }\end{array}$ & 2010 & - \\
\hline $\begin{array}{l}\text { température minimum (m } \\
\text { température maximum ( } \\
\text { température moyenne (mo } \\
\text { somme annuelle des préci } \\
\text { vitesse moyenne du vent }\end{array}$ & $\begin{array}{l}\text { inimum annuel des tempe } \\
\text { aximum annuel des temp } \\
\text { yenne annuelle des temp } \\
\text { bitations }\end{array}$ & $\begin{array}{l}\text { ratures journalières) } \\
\text { ratures journalières) } \\
\text { moyennes journalières) }\end{array}$ & & $\begin{array}{l}{ }^{\circ} \mathrm{C} \\
{ }^{\circ} \mathrm{C} \\
{ }^{\circ} \mathrm{C} \\
\text { millimètres } \\
\mathrm{km} / \mathrm{h}\end{array}$ \\
\hline $\begin{array}{l}\text { prix des terres et prés } \\
\text { libres de plus de } 70 \text { ha }\end{array}$ & $32 \mathrm{NRA}^{3}$ & Agreste & 2010 & $€$ courant/ha \\
\hline $\begin{array}{l}\text { données socio-économiques } \\
\text { densité de population } \\
\text { part d'agriculteurs } \\
\text { part de cadres } \\
\text { grand pôle urbain }\end{array}$ & communes & Insee & 2010 & $\begin{array}{l}- \\
\text { habitants } / \mathrm{km}^{2} \\
\% \\
\% \\
-\end{array}$ \\
\hline
\end{tabular}

Le tableau 2 donne la fréquence des différentes catégories d'usage des sols dans ces deux échantillons. Notons que pour 11 points Teruti-Lucas (tous situés dans l'échantillon test) l'usage des sols est manquant (nphys=9999 zones interdites, photos non interprétées).

TABLEAU 2 - Fréquence des catégories d'usage des sols en 2010 dans les deux échantillons (nombre de points Teruti-Lucas et \%)

\begin{tabular}{llrrrr|rr}
\hline landuse & libellé & \multicolumn{2}{c|}{ échantillon d'apprentissage } & \multicolumn{2}{c|}{ échantillon test } & \multicolumn{2}{|c}{ total } \\
& & effectif & 7,4 & 909 & 7,2 & 1842 & 7,3 \\
effectif & $\%$ & effectif & $\%$ \\
\hline 1 & usage urbain & 933 & 26,4 & 3252 & 25,7 & 6596 & 26,1 \\
2 & usage agricole & 3344 & 30,9 & 4051 & 32,0 & 7957 & 31,4 \\
3 & forêts & 3906 & 25,5 & 3279 & 25,9 & 6510 & 25,7 \\
4 & prairies & 3231 & 9,8 & 1155 & 9,1 & 2401 & 9,5 \\
5 & sols naturels & 1246 & 100 & 12646 & 100 & 25306 & 100 \\
\hline Total & & 12660 & & & & &
\end{tabular}

\subsection{Niveaux d'agrégation}

Comme évoqué dans l'introduction et dans la section 2.3, nous considérons plusieurs zonages du territoire en carreaux de façon à étudier la qualité d'estimation et de prédiction en fonction de la résolution finale. Pour cela, nous allons définir une série de maillages emboîtés obtenus par agrégations successives des carreaux du maillage précédent. 
Le niveau d'agrégation le plus fin, noté $A_{0}$, est constitué des points Teruti-Lucas tandis que le niveau le plus agrégé, noté $A_{7}$, est la région Midi-Pyrénées. Les niveaux intermédiaires sont des grilles. La grille initiale, notée $A_{1}$, a été conçue de façon à ce que chaque carreau contienne un segment Teruti-Lucas et un seul, afin de former un maillage ininterrompu du territoire. On parlera du niveau segments. Les carreaux de cette grille, dénommés ci-après " carreaux unités », sont centrés sur le barycentre des points d'un segment Teruti-Lucas et ont une taille de $4,2 \mathrm{~km}$ de côté. Cette grille comporte 2579 carreaux et est représentée dans la figure 3 .

Nous avons aussi construit des agrégations de ces « carreaux unités » afin d'obtenir un maillage de plus en plus grossier jusqu'à obtenir la région entière. A chaque étape, quatre carreaux sont réunis pour en former un seul. On obtient donc une deuxième grille (niveau d'agrégation $A_{2}$ ) dont chaque carreau est constitué de quatre « carreaux unités », puis une troisième (niveau d'agrégation $A_{3}$ ) dont chaque carreau est constitué de 16 « carreaux unités », etc. jusqu'à la dernière grille (niveau d'agrégation $A_{6}$ ) dont chaque carreau est constitué de 1024 « carreaux unités »(cf. tableau 3 ).

TABLEAU 3 - Caractéristiques des grilles

\begin{tabular}{lrrrr}
\hline Grille $\begin{array}{r}\text { Nombre de } \\
\text { « carreaux unités » } \\
\text { agrégés }\end{array}$ & $\begin{array}{r}\text { Superficie } \\
\text { approximative }\end{array}$ & $\begin{array}{r}\text { Nombre de } \\
\text { points } \\
\text { par carreau }\end{array}$ & $\begin{array}{r}\text { Nombre total } \\
\text { de carreaux }\end{array}$ \\
\hline$A_{1}$ & 1 & $18 \mathrm{~km}^{2}$ & 1 à 10 & 2579 carreaux \\
$A_{2}$ & 4 & $72 \mathrm{~km}^{2}$ & 1 à 40 & 689 carreaux \\
$A_{3}$ & 16 & $288 \mathrm{~km}^{2}$ & 4 à 160 & 192 carreaux \\
$A_{4}$ & 64 & $1152 \mathrm{~km}^{2}$ & 10 à 640 & 59 carreaux \\
$A_{5}$ & 256 & $4608 \mathrm{~km}^{2}$ & 184 à 2559 & 20 carreaux \\
$A_{6}$ & 1024 & $18432 \mathrm{~km}^{2}$ & 184 à 6605 & 8 carreaux \\
\hline
\end{tabular}

Cette famille de maillages étant définie, nous devons à présent expliquer comment définir les probabilités estimées ainsi que les prédictions à chaque niveau.

Pour agréger les estimations, la méthode standard dans cette situation, que ce soit pour les MNL ou les arbres, est de calculer tout d'abord une simple moyenne des probabilités estimées au niveau point sur l'ensemble des points d'un carreau donné. Pour définir par contre les prédictions au niveau agrégé, on a le choix entre deux solutions. La première est l'exact pendant de ce qu'on a fait au niveau des points : elle consiste, à partir des probabilités estimées agrégées, à prédire par l'usage correspondant à la probabilité estimée maximale. Nous proposons l'alternative suivante qui consiste à faire au niveau de chaque point un tirage aléatoire d'une multinomiale ayant pour paramètres les probabilités estimées au point et à en déduire ensuite au niveau du carreau les fréquences empiriques correspondant à chaque usage. Il est important de remarquer qu'il n'est plus possible, pour évaluer la qualité des prédictions, d'utiliser le taux de bien classés comme nous l'avions fait au niveau des points, car au niveau des carreaux, nous ne pouvons plus comparer la prédiction à une réalité.

\section{Résultats}

Dans cette partie, nous présentons les résultats d'ajustement et de prédiction des divers modèles. Il est important de noter que pour la première étape, il s'agit de l'estimation et de la prédiction au niveau des points Teruti-Lucas alors que pour la deuxième étape, il s'agit des estimations au niveau des carreaux. 


\subsection{Première étape : estimation et prédiction au niveau des points Teruti- Lucas}

Dans cette étape, les modèles sont ajustés sur les observations aux points Teruti-Lucas et les prédictions sont calculées à ce même niveau. Les modèles ajustés sont ceux présentés à la section 2: les modèles MNL et les arbres de classement. Nous partons d'un premier modèle multinomial logit dit «économétrique », noté MNL-E, pour lequel le choix des variables est motivé par la littérature. Selon la littérature économique empirique [Lubowski, 2002 sur l'utilisation des sols, les variables explicatives qui influent sur la décision de l'utilisation des sols incluent les rentes associées aux différents usages ou à défaut des proxy de ces rentes tels que les prix des inputs et des outputs, les aides publiques, la densité de la population (comme proxy de la rente urbaine), ainsi que d'autres variables pédoclimatiques telles que pente, altitude, qualité du sol, température, précipitations, etc.

Les résultats du modèle économétrique MNL-E présentés dans le tableau 16 montrent que les modalités de la variable couverture des sols CLC2 sont significatives pour déterminer les usages des sols. Le prix des terres agricoles est significatif et a un effet positif sur l'usage agricole, en forêt et prairies par rapport à l'usage urbain. La densité de la population n'a pas d'impact significatif sur les usages agricoles et forêt mais a un impact négatif et significatif sur les usages prairies et sols naturels. Un résultat qui peut paraître surprenant est la non-significativité des modalités de la variable qualité des sols. Une explication possible serait le fait que l'information contenue dans cette variable est déjà prise en compte dans la variable de couverture des sols CLC2 ainsi que dans la variable altitude. En ce qui concerne la qualité de prédiction du modèle MNL-E, le taux de points bien classés est de $65,12 \%$ ce qui est assez comparable aux autres modèles MNL-Cm et MNL-S (voir tableau 4).

TABleau 4 - Taux de bien-classés selon le modèle

\begin{tabular}{|c|c|c|}
\hline Variables restantes dans le modèle & MNL & arbres \\
\hline CLC2 + altitude & MNL-S : $65,15 \%$ & TREE-S : $64,92 \%$ \\
\hline $\begin{array}{l}\text { CLC } 2+\text { altitude }+ \text { MAT }+ \text { TEXT }+ \text { prixterre } \\
+ \text { cadre }\end{array}$ & - & TREE-Ct : \\
\hline $\begin{array}{l}\text { CLC } 2+\text { altitude }+ \text { tempmini }+ \text { ventmoy } \\
+ \text { tempmaxi }+ \text { altitude_lag }\end{array}$ & MNL-Cm : $65,37 \%$ & - \\
\hline $\begin{array}{l}\text { CLC2 + altitude + prixterre }+ \text { densite }+ \text { tempmini } \\
+ \text { tempmaxi }+ \text { tempmoy }+ \text { pluie }+ \text { TEXT }\end{array}$ & MNL-E : $65,12 \%$ & TREE-E : $65,04 \%$ \\
\hline
\end{tabular}

En cherchant à optimiser la prédiction (sélection du modèle par méthode pas-à-pas maximisant le taux de bien-classés dans l'échantillon test), on obtient un ensemble de variables explicatives pour le modèle MNL-Cm qui diffère de la sélection réalisée pour MNL-E (cf. tableau 17). En particulier les variables socio-économiques (prixterre, densité) et la texture du sol ne sont pas retenues. Les variables température moyenne et pluie disparaissent au profit du vent moyen et de l'altitude des voisins. Les six variables retenues sont CLC2, altitude, température minimum, vitesse du vent, température maximum et altitude dans le voisinage. Le taux de bien-classés est légèrement amélioré avec une valeur de $65,37 \%$ (voir tableau 4 ).

Concernant les arbres de classement, on utilise l'algorithme CART et l'ensemble des variables explicatives hormis celles mesurées dans le voisinage 5 . Si on utilise les paramètres par défaut de

5. Notons que si on introduit les variables de voisinage, d'une part l'altitude des voisins remplace l'altitude et, d'autre part, la part de cadres dans le voisinage est aussi sélectionné (en plus de la variable initiale), sans que cela améliore le taux de bien-classés qui devient 64,73\%. 
la fonction rpart, le critère de complexité vaut 0,01 et on obtient un arbre très élagué qui ne fait intervenir que deux variables explicatives (CLC2 et altitude). Pour obtenir un nombre de variables comparable à celui de MNL-Cm, nous fixons le critère de complexité initial à 0,0001 . Pour obtenir l'arbre TREE-Ct, nous utilisons la fonction rpart qui fournit un arbre maximal et la fonction prune qui permet d'élaguer l'arbre en utilisant la procédure décrite dans Breiman et al. [1984]. Avec cette méthode, on aboutit à un $c p=0,001$. L'arbre retenu est très complexe, il comporte 16 nouds et prend en compte 6 variables (CLC2, altitude, prix des terres, part de cadres, matériau de base et texture du sol). Les variables météo n'interviennent plus mais on retrouve des variables socio-économiques (prix des terres et part de cadres) et portant sur la nature du sol (la texture, comme dans MNL-E, et le matériau de base). En raison de sa complexité, il ne sera pas détaillé davantage. Le taux de bien-classés est de 64,83 \%, soit légèrement inférieur à ceux de MNL-E et MNL-Cm (voir tableau 4).

A partir de cet arbre, on cherche à maximiser le taux de bien-classés en élaguant progressivement l'arbre (cf. tableau 5). Le meilleur taux de bien-classés, égal à 64,92 \%, est obtenu avec l'arbre TREE-S qui comporte 10 nœuds et seulement deux variables : CLC2 et altitude (cf. figure 4).

TABLEAu 5 - Evolution du taux de bien-classés selon le critère de complexité de l'arbre

\begin{tabular}{cccc}
\hline$c p$ & nombre de nœuds & taux de bien-classés & \\
\hline 0,001 & 16 & $64,83 \%$ & (TREE-Ct) \\
0,002 & 14 & $64,90 \%$ & \\
0,0025 & 13 & $64,87 \%$ & \\
0,003 & 11 & $64,92 \%$ & \\
0,005 & 10 & $64,92 \%$ & (TREE-S) \\
0,007 & 8 & $64,48 \%$ & \\
0,008 & 7 & $64,15 \%$ & \\
0,01 & 6 & $63,47 \%$ & \\
\hline
\end{tabular}

Enfin si on construit un MNL uniquement avec les variables de l'arbre TREE-S (CLC2 et altitude), modèle MNL-S (cf. tableau 18), on obtient un taux de bien-classés de 65,15\%, équivalent à celui du modèle économétrique MNL-E et à peine inférieur à celui du modèle MNL-Cm (voir tableau 4).

La principale différence entre les modèles MNL-S et MNL-E est la non significativité du coefficient de la variable altitude pour l'usage agricole dans le modèle économétrique alors que ce coefficient est significatif à 1 pour 1000 dans le modèle simple.

Le tableau 6 regroupe des indicateurs de qualité pour les trois modèles de régression logistique multinomiale présentés. Nous voyons que les trois modèles fournissent des résultats très similaires.

TABLEAU 6 - Comparaison de la qualité des modèles de régression logistique multinomiale

\begin{tabular}{llll}
\hline Indicateur & MNL-E & MNL-Cm & MNL-S \\
\hline BIC & 26255,1 & 26197,3 & 26197,1 \\
AIC & 25540,3 & 25661,2 & 25780,2 \\
$R^{2}$ de Mc Fadden & 0,3251 & 0,3206 & 0,3166 \\
taux de bien-classés & $65,12 \%$ & $65,37 \%$ & $65,15 \%$ \\
\hline
\end{tabular}




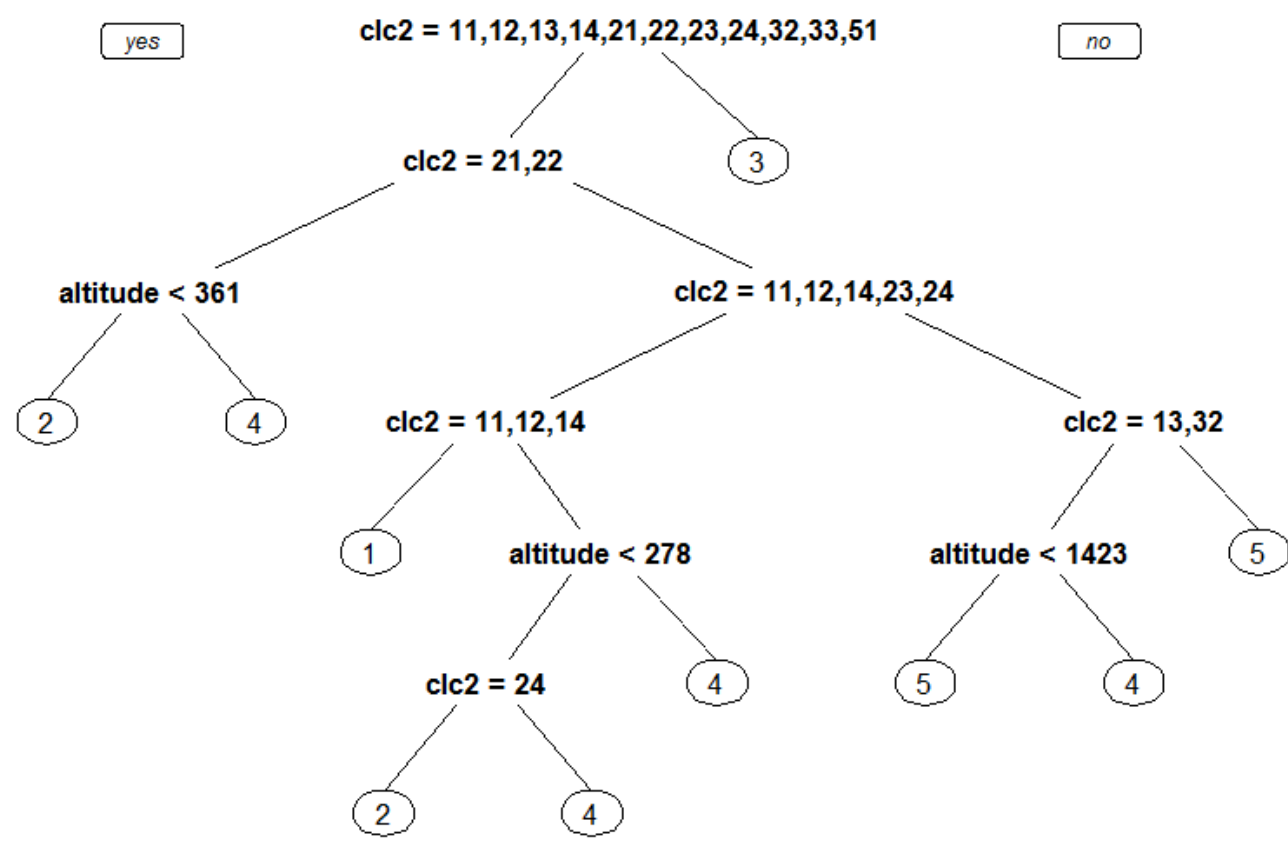

1 urbain $\quad C L C 2=($ « Zones urbanisées », «Zones industrielles ou commerciales et réseaux de communication » ou « Espaces verts artificialisés, non agricoles »)

2 agricole $\quad$ a. $C L C 2=($ « Terres arables » ou « Cultures permanentes ») et altitude $<361 \mathrm{~m}$

b. $C L C 2=($ (Zones agricoles hétérogènes ») et altitude $<278 \mathrm{~m}$

3 forêts $\quad C L C 2=($ « Forêts » ou « Zones humides intérieures »)

4 prairies a. $C L C 2=($ « Terres arables » ou « Cultures permanentes ») et altitude $\geq 361 \mathrm{~m}$

b. $C L C 2=«$ Prairies » et altitude $<278 \mathrm{~m}$

c. $C L C 2=($ «rairies » ou « Zones agricoles hétérogènes ») et altitude $\geq 278 \mathrm{~m}$

d. $C L C 2=$ ( « Mines, décharges et chantiers », « Milieux à végétation arbustive et/ou herbacée $»)$ et altitude $\geq 1423 \mathrm{~m}$

5 sols naturels a. $C L C 2=($ «Mines, décharges et chantiers », « Milieux à végétation arbustive et/ou herbacée ») et altitude $<1423 \mathrm{~m}$

b. $C L C 2=($ Espaces ouverts, sans ou avec peu de végétation » ou « Eaux continentales »)

Avec près de deux tiers de points dont l'usage des sols est correctement prédit, les modèles sont très significativement meilleurs qu'un classement aléatoire, selon la statistique $Q_{P R E S S}$. On remarque toutefois qu'on ne retrouve pas la variabilité observée d'usages de sol au niveau segment dans nos prédictions. Alors que $67 \%$ des segments ont au moins trois usages observés différents, cette proportion tombe à 14,7\% pour les usages prédits. La figure 5 illustre aussi ce point.

Par ailleurs, même si nous ne présentons pas les résultats, les taux de bien-classés obtenus pour les points de l'échantillon d'apprentissage et pour les points de l'échantillon test sont très similaires, ce qui montre la stabilité de nos modèles. Enfin il est intéressant de remarquer que les risques empiriques de Bayes (qui valent 1 moins la moyenne empirique des probabilités maximales estimées) sont pratiquement égaux aux taux de mal-classés, soit autour de $35 \%$. Il paraît donc difficile d'améliorer ces modèles au niveau ponctuel. Mais on peut essayer d'améliorer la qualité de prédiction en considérant des niveaux spatiaux plus agrégés. Cela fait l'objet de la section suivante. 
FiguRE 5 - Nombre d'usages de sol différents par segment, usages observés (carte de gauche) ou prédits par le modèle MNL-Cm (carte de droite)
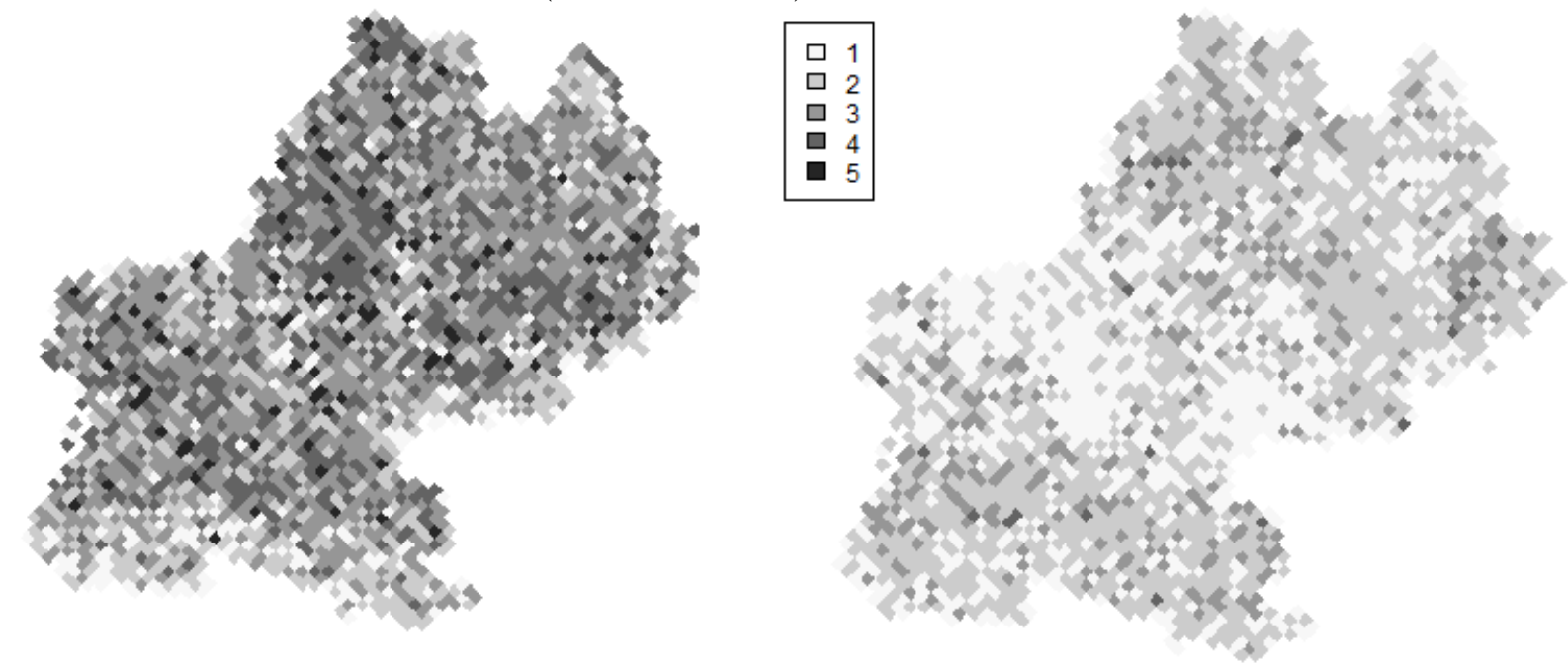

\subsection{Deuxième étape : estimation au niveau des carreaux}

L'objectif final est d'obtenir des prédictions ou des probabilités estimées de l'usage des sols sur un carroyage du territoire qu'il va ensuite utiliser soit pour cartographier soit comme ingrédient dans un modèle. Selon le cas, il peut avoir à se poser la question du choix de la taille de ce carroyage. Dans tous les cas, il va devoir agréger sur les carreaux les probabilités estimées obtenues au niveau des points lors de la première étape. Nous abordons ces deux questions dans cette partie.

Pour mesurer la qualité de prédiction ou d'estimation des probabilités, nous utilisons le score de Brier pondéré défini à la section 2.3. Les niveaux d'agrégation $A_{0}$ à $A_{7}$ sont définis à la section 3.4. $A_{0}$ dénote le niveau des points Teruti-Lucas, $A_{1}$ le niveau des segments, $A_{2}$ à $A_{6}$ les grilles successives et $A_{7}$ le niveau global de la région Midi-Pyrénées.

Les figures 6 à 8 représentent les scores de Brier pondérés en fonction du niveau d'agrégation, selon trois méthodes de prédiction ou d'estimation des probabilités pour la première figure et selon différents modèles pour les deux figures suivantes. La premières des trois méthodes que nous comparons dans la figure 6 consiste à utiliser les probabilités estimées au niveau point et à les agréger aux niveaux supérieurs. Les deux autres méthodes, détaillées à la section 3.4, sont basées sur une prédiction au niveau point et conduisent à des probabilités agrégées aux niveaux supérieurs. Sur l'ensemble de ces figures, on remarque un score plutôt élevé au niveau individuel $\left(A_{0}\right)$, beaucoup plus faible au niveau segments $\left(A_{1}\right)$, encore un peu plus faible au niveau $A_{2}$ (où un carreau contient quatre segments) et qui se stabilise en général à partir du niveau $A_{3}$ (16 segments) pour finir comme attendu à zéro au niveau de la région $\left(A_{7}\right)$ pour les modèles logit.

La figure 6 montre que l'utilisation des probabilités estimées conduit à un score de Brier plus faible que les deux méthodes d'agrégation des prédictions. Au niveau individuel, le tirage multinomial est particulièrement mauvais mais dépasse la probabilité maximale dès le niveau segments et est comparable au score obtenu avec les probabilités estimées à partir du niveau $A_{2}$.

La figure 7 montre que les modèles sélectionnés à la section 4 conduisent à des scores de Brier complètement équivalents pour chacun des niveaux d'agrégation spatiale envisagés.

La figure 8 propose une comparaison des scores de Brier pondérés entre le modèle MNL-Cm et des modèles simplifiés. On voit clairement que le modèle qui ne prend en compte que la variable 
Figure 6 - Scores de Brier pondérés pour le modèle MNL-Cm selon la méthode de prédiction

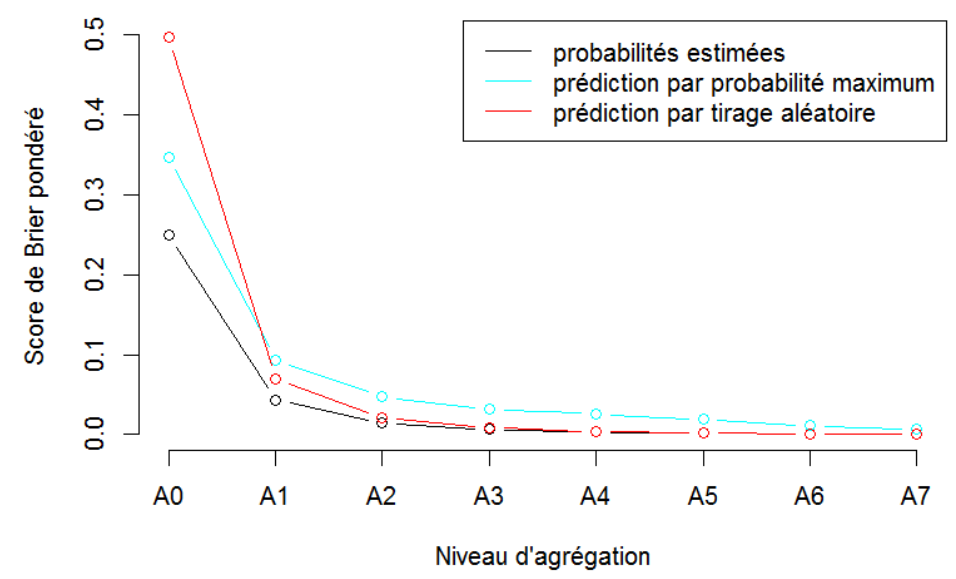

Figure 7 - Scores de Brier pondérés selon le modèle (méthode : probabilités estimées)

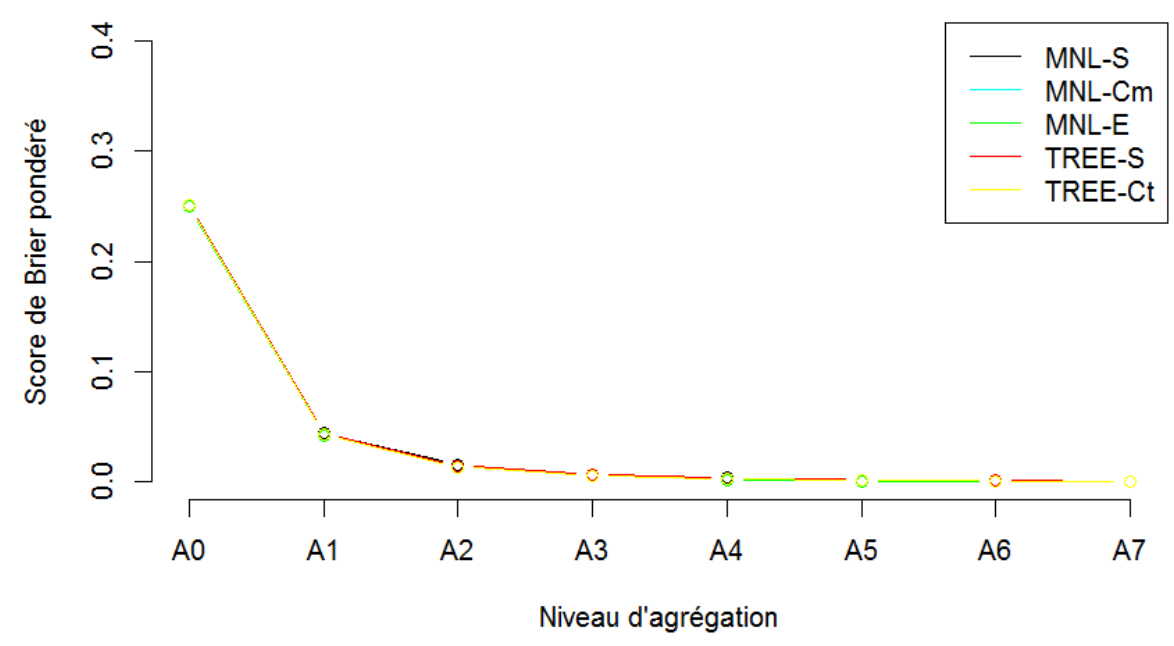

CLC2 donne des résultats très similaires aux résultats du modèle MNL-Cm. Peu importe le niveau d'agrégation, en termes de qualité de prédiction, il n'est pas utile d'introduire d'autres variables que l'occupation du sol mesurée par Corine Land Cover au niveau 2 (15 catégories). De manière symétrique, les autres variables du modèle économétrique sans CLC2 mènent à une qualité de prédiction comparable à CLC2 à partir du niveau $A_{3}$. Au contraire, les Corine Land Cover au niveau 1 (5 catégories) ne permettent pas d'atteindre une qualité équivalente aux autres modèles quel que soit le niveau d'agrégation (sauf au niveau global par propriété des MNL).

Les figures 9 et 10 permettent d'analyser le gain relatif en termes de score de Brier pondéré lors des étapes d'agrégation successives. Précisément on calcule la différence entre le score aux niveaux $A_{l-1}$ et $A_{l}$ rapportée au score individuel. Ainsi pour le modèle MNL-Cm, ce ratio vaut 0,826 lorsque l'on passe du niveau individuel au niveau segments, ce qui signifie que l'on élimine $83 \%$ de la valeur du score de Brier pondéré en agrégeant au segment. On note que la courbe n'est pas nécessairement décroissante. En particulier pour le modèle avec CLC1 seulement, le gain relatif augmente à la fin ce qui est dû au fait que la somme de ces ratios est égale à 1.Ce graphique est une aide à la décision pour le choix d'un niveau d'agrégation. Dans notre exemple, il montre qu'il faut agréger au niveau segments, ou davantage, car cette première agrégation apporte un 
Figure 8 - Scores de Brier pondérés selon le modèle, modèles moins « bons » (méthode : probabilités estimées)

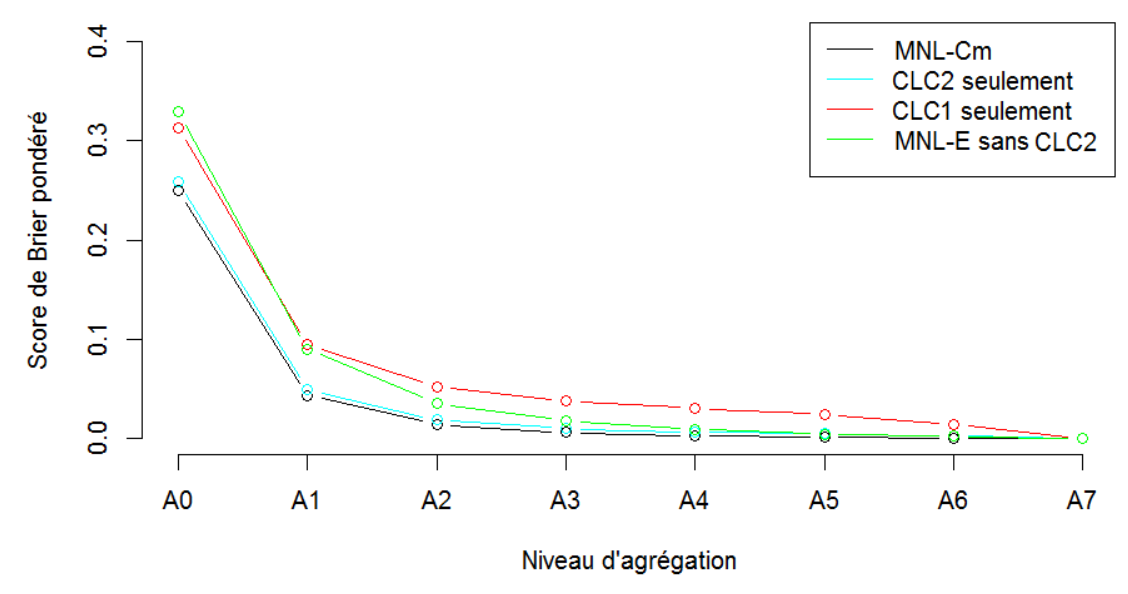

Figure 9 - Apport de l'agrégation selon le modèle (score de Brier pondéré total, méthode : probabilités estimées)

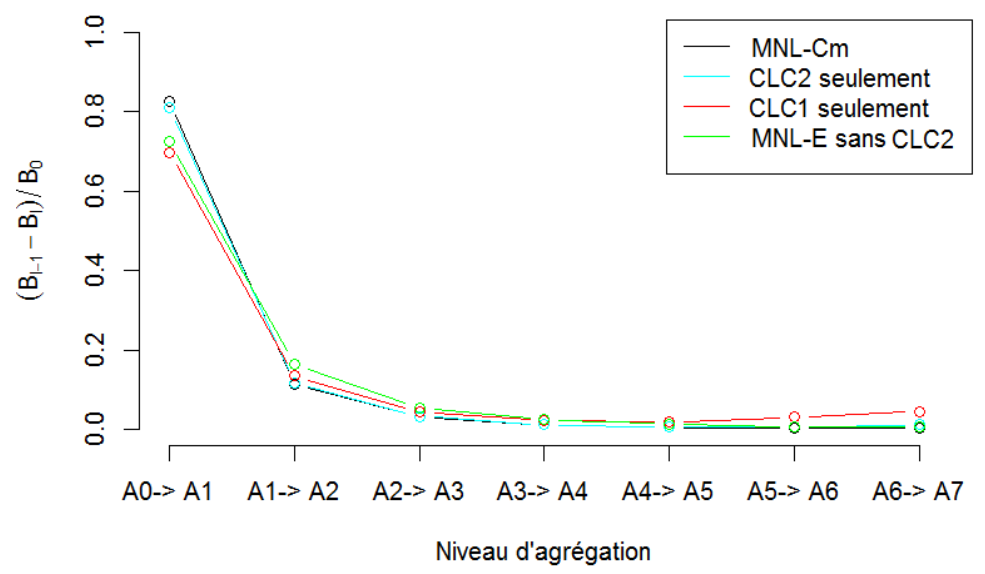

gain majeur en termes de qualité de prédiction tandis que les agrégations suivantes n'apportent pas un gain notable.

Notons qu'on peut interpréter les valeurs du ratio en termes de diminution d'erreur moyenne d'estimation des probabilités en remarquant que la racine carrée du double du score de Brier correspond à l'erreur quadratique moyenne qui est du même ordre que l'erreur moyenne en valeur absolue. Ainsi, pour un gain de 0,826 , on peut calculer $\sqrt{2 \times(1-0,826)}=0,59$ et dire que l'erreur moyenne en valeur absolue est diminuée de $41 \%$ en agrégeant du point au segment.

Il est possible comme dans la figure 10 de décomposer les ratios selon les différents usages des sols. Dans notre exemple, il n'y a pas de différence notable entre les différents usages et il faut toujours agréger au niveau segments pour obtenir une bonne qualité de prédiction. 
Figure 10 - Apport de l'agrégation selon l'usage des sols (score de Brier pondéré par usage, méthode : probabilités estimées)

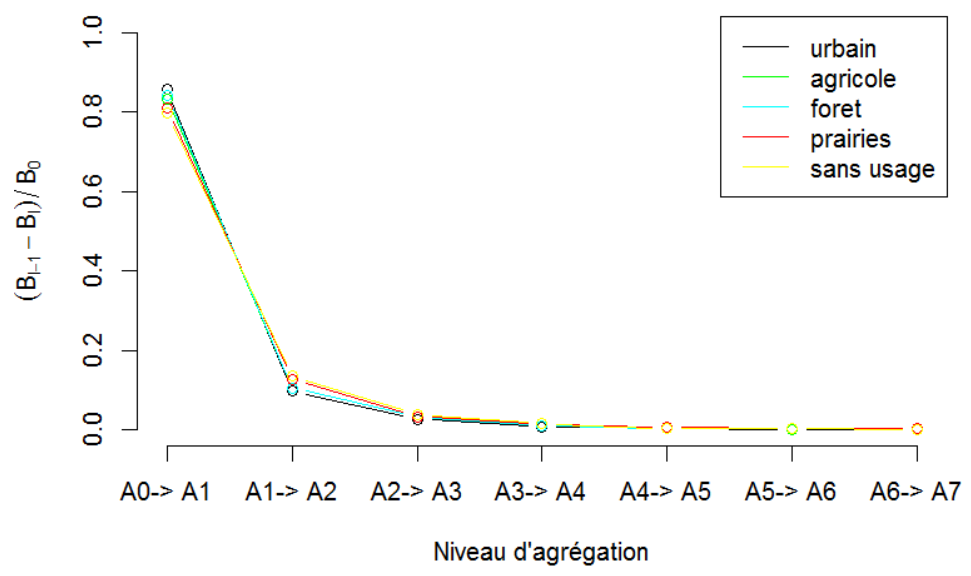

\section{Conclusion}

Notre objectif était de modéliser l'usage des sols en se restreignant à des covariables facilement disponibles, sur Internet ou sur demande auprès de fournisseurs de données. Il est reconnu qu'une des difficultés de la modélisation des usages des sols au niveau individuel est le manque fréquent de «bonnes» variables explicatives ou leur incompatibilité d'échelle, en particulier pour les variables économiques (rentes, coûts de conversion et les prix) Chakir, 2015. Nous avons été confrontés à ces difficultés autant pour les variables socio-économiques, qui n'étaient disponibles qu'au niveau communal ou à celui des NRA (Nouvelles Régions Agricoles), que pour les variables météorologiques (dont la résolution n'était que de $25 \times 25 \mathrm{~km}$ ). Nos analyses montrent le fort pouvoir explicatif de deux variables très simples, l'occupation des sols mesurée par Corine Land Cover (au niveau 2, soit en 15 catégories) et l'altitude. Ces deux variables sont retenues dans l'ensemble des modèles étudiés. Rappelons qu'à elles seules, elles conduisent à un taux de bien-classés de 65,15\% (modèle de régression logistique multinomiale dit « simple »). L'ajout de variables supplémentaires (météorologiques, socio-économiques ou biophysiques) n'améliore que très marginalement les taux de bien-classés.

Deux autres résultats nous semblent importants à souligner. Tout d'abord, les modèles MNL testés donnent systématiquement des résultats légèrement meilleurs que les arbres de classement équivalents (c'est-à-dire avec le même sous-ensemble de prédicteurs) en termes de taux de bienclassés. Ensuite, nous remarquons une grande stabilité des résultats, en particulier une robustesse des arbres de classement, qui n'est pas classique dans la littérature, mais qui provient du fait que la variable CLC2 est une variable qualitative qui joue un rôle prépondérant dans les résultats.

Plusieurs pistes de recherche peuvent être envisagées à la suite de ce travail.

Premièrement, face à la difficulté de sélection de variables en présence d'un grand nombre de variables qualitatives ayant de nombreuses modalités, les méthodes de type lasso peuvent être envisagées. Ces méthodes ont l'avantage d'utiliser une pénalisation pour combiner la phase d'estimation à celle du choix d'un modèle parcimonieux dans le cas des MNL Meier et al., 2008, Tutz et al. 2015.

Deuxièmement, la qualité de prédiction des modèles logit multinomiaux pourrait être améliorée en y introduisant explicitement l'auto-corrélation spatiale. L'estimation des modèles de choix discret avec de l'auto-corrélation spatiale reste un défi au niveau des calculs. Les méthodes d'estimation développées par Ferdous et Bhat [2013]; Sidharthan et Bhat [2012] semblent être prometteuses et présentent une bonne alternative aux méthodes bayésiennes ou aux méthodes d'estimation par 
simulation qui restent assez intensives en termes de calculs.

Troisièmement, nos résultats montrent que la qualité de prédiction au point s'améliore significativement lorsque les résultats sont agrégés au niveau des carreaux. Ceci peut poser la question sur la pertinence de l'estimation du modèle d'usage des sols au niveau des points Teruti-Lucas. L'utilisation du score de Brier pondéré permet toutefois de montrer qu'une agrégation au niveau des segments Teruti-Lucas est suffisante pour améliorer les résultats et que des niveaux d'agrégation supérieurs ne sont pas nécessaires. Une fois défini un niveau d'agrégation adéquat, on pourrait aussi chercher à estimer un modèle agrégé de type land use share Chakir et Le Gallo, 2013. La variable d'intérêt devient alors la proportion de chaque usage de sol dans l'unité géographique agrégée, variable de type donnée de composition ( $\mathrm{CoDa}$ ) dont le traitement nécessite une méthodologie adaptée Aitchison, 2003.

Enfin, concernant les implications en termes de politiques économiques des usages des sols, aucune législation claire n'existe à ce jour pour la protection des sols en France. Ainsi, le sol est souvent mentionné indirectement à travers des textes sur la réglementation de l'urbanisation (loi ALUR), l'évaluation des incidences environnementales (les lois Grenelle 1 et 2 ) ou les réformes des aides agricoles (La loi de modernisation de l'agriculture). Les effets des politiques de zonage ne sont donc pas dissociables des politiques agricoles ou des politiques d'aménagement du territoire Facchini, 2000. Il est important d'étudier l'interaction entre usages des sols de manière exhaustive pour mieux comprendre les enjeux. Dans ce contexte, le développement d'outils de modélisation, tels que ceux proposés dans ce papier, présente une première étape de prédiction des usages des sols à partir de variables facilement accessibles. Cette première étape méthodologique ouvre de nouvelles voies de recherche pour examiner les implications des usages des sols dans le domaine de l'environnement (biodiversité, qualité de l'eau, qualité du sol, qualité de l'air) ou de l'aménagement du territoire (analyse de l'artificialisation des sols, extension des villes, suivi des espaces protégés).

\section{Remerciements}

Ce travail a été partiellement financé par l'Agence Nationale de la Recherche à travers le projet ModULand (ANR-11-BSH1-005). Nous remercions le Service de la Statistique et de la Prospective du Ministère de l'Agriculture pour avoir mis à notre disposition les données de l'enquête TerutiLucas dans le cadre du projet ANR ModULand, l'Unité INFOSOL de l'INRA pour les données pédologiques (BDGSF) et le Joint Research Center-MARS de la Commission Européenne pour les données météorologiques (Interpolated Meteorological Data Base). Nous remercions également Antoine Lacroix pour le travail préparatoire accompli lors de son stage de M1. 


\section{Références bibliographiques}

Aitchison J. [2003]. A concise guide to compositional data analysis. Dans 2nd Compositional Data Analysis Workshop, .

Akaike H. [1974]. «A new look at the statistical model identification ». Automatic Control, IEEE Transactions on, 19(6), 716-723.

Breiman L., Friedman J., Stone C. et Olshen R. [1984]. Classification and Regression Trees. The Wadsworth and Brooks-Cole statistics-probability series. Taylor \& Francis.

Brier G. W. [1950]. «Verification of forecasts expressed in terms of probability ». Monthly weather review, 78(1), 1-3.

Buja A., Stuetzle W. et Shen Y. [2005]. « Loss functions for binary class probability estimation and classification : Structure and applications ». Working draft, November.

Chakir R. [2015]. « L'espace dans les modèles économétriques d'utilisation des sols : enjeux méthodologiques et applications empiriques ». Revue d'Economie Rurale et Urbaine, à paraître.

Chakir R. et Le Gallo J. [2013]. « Predicting land use allocation in France : A spatial panel data analysis ». Ecological Economics, 92(0), 114-125.

Chakir R. et Parent O. [2009]. « Determinants of land use changes : A spatial multinomial probit approach ». Papers in Regional Science, 88(2), 327-344.

Chomitz K. M. et Gray D. A. [1996]. « Roads, land use, and deforestation : a spatial model applied in Belize ». World Bank Economic Review, 10(3), 487-512.

Domencich T. A. et McFadden D. [1975]. Urban Travel Demand-A Behavioral Analysis. NorthHolland Publishing Co.

Facchini F. [2000]. Politique agricole, zonage et aménagement du territoire rural. Revue d'Economie Régionale et Urbaine, (1), 24-47.

Ferdous N. et Bhat C. [2013]. « A spatial panel ordered-response model with application to the analysis of urban land-use development intensity patterns ». Journal of Geographical Systems, 15(1), 1-29.

Hair J. [2010]. Multivariate data analysis : A global perspective. Global Edition. Pearson Education.

Hastie T., Tibshirani R. et Friedman J. [2009]. The Elements of Statistical Learning. Data Mining, Inference, and Prediction. Springer Series in Statistics. Springer.

Irwin E. G. et Geoghegan J. [2001]. « Theory, data, methods : developing spatially explicit economic models of land use change ». Agriculture, Ecosystems \& Environment, 85(1), 7-23.

Irwin E. G. et Wrenn D. H. [2014]. « An assessment of empirical methods for modeling land use ». Dans Duke, J. M. et Wu J. The Oxford Handbook of Land Economics, 327-351. Oxford University Press, New York.

Jobson J. [1999]. Applied multivariate data analysis : regression and experimental design. Springer Texts in Statistics. Springer New York.

Lambin E. F., Rounsevell M. et Geist H. [2000]. « Are agricultural land-use models able to predict changes in land-use intensity?». Agriculture, Ecosystems \& Environment, 82(1), 321-331. 
Lubowski R. N. [2002]. "Determinants of land-use transitions in the United States : Econometric analysis of changes among the major land-use categories ". Thèse de doctorat, Harvard University, Cambridge.

McFadden D. [1974]. "Conditional logit analysis of qualitative choice behavior ». Dans Zarembka P. Frontiers in Econometrics. Academic Press, New York.

McMillen D. P. [1989]. « An empirical model of urban fringe land use ». Land Economics, 138-145.

Meier L., Van De Geer S. et Bühlmann P. [2008]. " The group lasso for logistic regression ». Journal of the Royal Statistical Society : Series B (Statistical Methodology), 70(1), 53-71.

Merkle E. C. et Steyvers M. [2013]. « Choosing a strictly proper scoring rule ». Decision Analysis, 10(4), 292-304.

Munroe D. K. et Müller D. [2007]. « Issues in spatially explicit statistical land-use/cover change (LUCC) models : Examples from western Honduras and the Central Highlands of Vietnam ». Land use policy, 24(3), 521-530.

Munroe D. K., Southworth J. et Tucker C. M. [2004]. «Modeling spatially and temporally complex land-cover change : The Case of Western Honduras ». The Professional Geographer, $\mathbf{5 6}(4), 544-559$.

Nelson G. C. et Hellerstein D. [1997]. « Do roads cause deforestation? Using satellite images in econometric analysis of land use ». American Journal of Agricultural Economics, 80-88.

Schwarz G. [1978]. « Estimating the Dimension of a Model ». Ann. Statist., 6(2), 461-464.

Shmueli G. [2010]. « To explain or to predict?». Statistical science, 289-310.

Sidharthan R. et Bhat C. R. [2012]. « Incorporating Spatial Dynamics and Temporal Dependency in Land Use Change Models ». Geographical Analysis, 44(4), 321-349, p. 321-349.

Team R. C. [2014]. $R$ : A language and environment for statistical computing. R Foundation for Statistical Computing, Vienna, Austria. URL http://www.R-project.org/.

Therneau T., Atkinson B. et Ripley B. [2014]. rpart : Recursive partitioning and regression trees. R package version 4.1-8, URL http://CRAN. R-project.org/package=rpart.

Train K. E. [2009]. Discrete Choice Methods with Simulation. Cambridge Books. Cambridge University Press.

Tufféry S. [2010]. Data mining et statistique décisionnelle : L'intelligence des données. Editions Technip.

Tutz G., Pößnecker W. et Uhlmann L. [2015]. « Variable selection in general multinomial logit models ». Computational Statistics \& Data Analysis, 82(0), 207 - 222.

Veldkamp A. et Lambin E. F. [2001]. « Predicting land-use change ». Agriculture, ecosystems \& environment, 85(1), 1-6.

Verburg P. H., Schot P. P., Dijst M. J. et Veldkamp A. [2004]. " Land use change modelling : current practice and research priorities ». GeoJournal, 61, 309-324.

Winkler R. L., Munoz J., Cervera J. L., Bernardo J. M., Blattenberger G., Kadane J. B., Lindley D. V., Murphy A. H., Oliver R. M. et Ríos-Insua D. [1996]. « Scoring rules and the evaluation of probabilities ». Test, $\mathbf{5}(1), 1-60$.

Zhiyu W. et Hasan A. [2014]. mnlogit : Multinomial logit model. R package version 1.1.1, URL http://CRAN.R-project.org/package=mnlogit 


\section{Annexes}

\subsection{Variable à expliquer}

TABLEAU 7: Correspondance entre la nomenclature de l'usage des sols en 57 postes (variable $N P H Y S$ ) et le regroupement en cinq catégories utilisé ici (variable landuse)

\begin{tabular}{lll} 
landuse & NPHYS & Libellé \\
\hline 1 & 11100 & Volumes construits bas \\
1 & 11200 & Volumes construits hauts \\
1 & 12100 & Sols de forme aréolaire revêtus ou stabilisés \\
1 & 12200 & Sols de forme linéaire revêtus ou stabilisés \\
1 & 13000 & Sols enherbés artificialisés \\
1 & 14200 & Sols nus artificialisés \\
2 & 11300 & Serres et abris hauts \\
2 & 13100 & Sols enherbés liés à la production agricole, hors élevage \\
2 & 14100 & Sols nus liés à une activité agricole \\
2 & 21100 & Blé tendre et épeautre \\
2 & 21200 & Blé dur \\
2 & 21300 & Orge et escourgeon \\
2 & 21500 & Avoine \\
2 & 21600 & Maïs \\
2 & 21820 & Triticale \\
2 & 21900 & Autres céréales \\
2 & 22100 & Pomme de terre \\
2 & 22200 & Betterave industrielle \\
2 & 22300 & Autres racines et tubercules \\
2 & 23110 & Tournesol \\
2 & 23120 & Colza et navette \\
2 & 23190 & Autres cultures industrielles oléagineuses \\
2 & 23200 & Cultures industrielles textiles \\
2 & 23320 & Pois sec protéagineux et pois fourrager \\
2 & 23330 & Fèves et fèveroles \\
2 & 23390 & Autres cultures industrielles annuelles \\
2 & 24100 & Légumes \\
2 & 24300 & Fleurs, plantes ornementales et pépinières toutes espèces \\
2 & 26000 & Jachère \\
2 & 27500 & Vigne \\
2 & 27100 & Pommiers \\
2 & 27200 & Autres fruitiers \\
2 & 27900 & Autres cultures permanentes \\
2 & 28000 & Jardins familiaux \\
3 & 31100 & Forêts de feuillus \\
3 & 31200 & Forêts de résineux \\
3 & 31300 & Forêts mixtes \\
3 & 31400 & Peupleraies en plein \\
3 & 32000 & Bosquets \\
3 & 33000 & Haies et alignements d'arbres \\
\hline & 34000 & Sols boisés à peuplement indéterminé (coupe rase) \\
\hline & 25100 & Fourrages annuels \\
\hline &
\end{tabular}




\begin{tabular}{lll}
\hline landuse & NPHYS & Libellé \\
\hline 4 & 25200 & Prairies temporaires semées essentiellement de graminées \\
4 & 25300 & Prairies temporaires semées essentiellement de légumineuses \\
4 & 25400 & Prairies permanentes productives \\
4 & 25500 & Prairies permanentes peu productives \\
4 & 25600 & Alpages \\
5 & 40000 & Landes, friches, maquis, garrigues, savanes \\
5 & 41000 & Superficies enherbées naturelles \\
5 & 60100 & Dune, plage \\
5 & 60200 & Rochers, éboulis \\
5 & 60300 & Sols nus naturels \\
5 & 70100 & Eaux intérieures \\
5 & 70200 & Plans d'eau côtiers \\
5 & 70300 & Glaciers, neiges éternelles \\
5 & 70400 & Zones humides \\
. & 88888 & Hors territoire \\
. & 99999 & Zones interdites, photos non interprétées \\
\hline
\end{tabular}

\subsection{Compléments sur les variables explicatives}

Occupation des sols : Corine Land Cover 2006 Nom de l'enquête : Corine Land Cover Promoteur : Agence européenne pour l'environnement, en France : Service de l'observation et des statistiques du ministère chargé de l'environnement

Disponibilité des données : gratuit ${ }^{6}$ après remplissage d'un formulaire

Echelle : 1 / 100000

La base CORINE Land Cover est une base de données européenne d'occupation biophysique des sols, produite dans le cadre du programme européen CORINE de coordination de l'information sur l'environnement. Le producteur pour la France est le Service de l'observation et des statistiques du ministère chargé de l'environnement. Les données sont issues de l'interprétation visuelle d'images satellitaires, avec des données complémentaires d'appui (en particulier les BD ORTHO et CARTO de l'IGN). L'occupation biophysique du sol prévaut à son utilisation, ainsi la nature des objets (forêts, cultures, surfaces en eau, roches affleurantes...) est privilégiée par rapport à leur fonction socio-économique (agriculture, habitat...).

«L'unité spatiale au sens de CORINE Land Cover est une zone dont la couverture peut être considérée comme homogène, ou être perçue comme une combinaison de zones élémentaires qui représente une structure d'occupation. La surface de la plus petite unité cartographiée (seuil de description) est de 25 ha.» Les polygones constituant les zones CLC ont des aires comprises entre 0,25 et $3857 \mathrm{~km}^{2}$ en Midi-Pyrénées, avec une moyenne de 2,6 $\mathrm{km}^{2}$. Pour plus d'informations se reporter au site Internet : http://www.statistiques.developpement-durable.gouv.fr/ donnees-ligne/t/methode-production-base-donnees.html?tx_ttnews\%5Btt_news\%5D=11268\& cHash=88595af0806f $46 f 2$ c8901fd438ea809f

Dans cette analyse, les codages aux niveaux 1 et 2 (respectivement en 5 et 15 catégories) de cette variable sont utilisés (cf. tableau 8).

6. http://www.statistiques.developpement-durable.gouv.fr/donnees-ligne/li/1825/1097/ occupation-sols-corine-land-cover.html 
TABleau 8 - Codage de la variable d'occupation des sols, Corine Land Cover, niveaux 1 et 2

\begin{tabular}{ll}
\hline CLC1 & CLC2 \\
\hline 1 Territoires artificialisés & 11 Zones urbanisées \\
& 12 Zones industrielles ou commerciales et réseaux de communication \\
& 13 Mines, décharges et chantiers \\
& 14 Espaces verts artificialisés, non agricoles \\
\hline 2 Territoires agricoles & 21 Terres arables \\
& 22 Cultures permanentes \\
& 23 Prairies \\
& 24 Zones agricoles hétérogènes \\
& 31 Forêts et milieux semi-naturels \\
& 32 Milieux à végétation arbustive et/ou herbacée \\
& 33 Espaces ouverts, sans ou avec peu de végétation \\
& 41 Zones humides intérieures \\
& 42 Zones hum. maritimes \\
\hline Z Zones humides & 51 Eaux continentales \\
& 52 Eaux maritimes
\end{tabular}

Nature du sol : Base de données géographique des sols de France (BDGSF) Nom de l'enquête : Base de données géographique des sols de France

Promoteur : INRA, Unité INFOSOL, Orléans

Année : 1998

Disponibilité des données : disponible sur demande pour un an (sur CD), 50 euros

Echelle : 1 / 1000000

« La BDGSF est une représentation simplifiée de la diversité spatiale de la couverture de sol. La méthodologie utilisée pour différencier et nommer les principaux types de sol est basée sur la terminologie de la légende de la carte des sols du monde établie en 1974 par la FAO à l'échelle du 1 / 5000 000. Cette terminologie est basée sur la distinction des processus pédologiques responsables de la différenciation des sols, c'est-à-dire la brunification, le lessivage, la podzolisation, l'hydromorphie, etc. Elle a été revue et adaptée pour prendre en compte les spécificités des paysages français. » Source : site Internet de la BDGSF http://www.gissol.fr/programme/bdgsf/ bdgsf.php.

Les Unités Cartographiques de Sols (UCS) sont des regroupements d'Unités Typologiques de Sols (UTS) qui sont localisables dans l'espace tandis qu'il n'est pas possible de localiser et de délimiter toutes les UTS à l'échelle de travail. Les polygones constituant les UCS ont des aires comprises entre 1,8 et $4369 \mathrm{~km}^{2}$ en Midi-Pyrénées. Pour plus d'informations se reporter au site Internet : http://www.gissol.fr/programme/bdgsf/contenu.php

Climat : European Climate Database site Internet : http://www.marsop.info http://marswiki.jrc.ec.europa.eu/agri4castwiki/index.php/Meteorological_data_froln_ ground_stations

Nom de l'enquête : Agri4cast Interpolated Meteorological database

Promoteur : JRC-MARS (Joint Research Center, Monitoring Agricultural ResourceS (MARS)

Unit, European Commission)

Années : 1975 à 2012

Disponibilité des données : enregistrement requis 
Nouvelles Régions Agricoles Les Nouvelles Régions Agricoles sont des regroupements de petites régions agricoles (PRA) effectués par l'Agreste. Les régions agricoles (RA) et PRA avaient été définies en 1946 pour mettre en évidence des zones agricoles homogènes. Une RA couvre un nombre entier de communes formant une zone d'agriculture homogène; une PRA est constituée par le croisement du département et de la RA. Dans la région Midi-Pyrénées, il y a 61 PRA et 32 NRA. L'aire des NRA est comprise entre 302,2 et $4284 \mathrm{~km}^{2}$, avec une moyenne de $1426 \mathrm{~km}^{2}$.

Tableau 9 - Statistiques descriptives de l'aire $\left(\mathrm{en}^{2} \mathrm{~km}^{2}\right)$ des polygones des différentes bases

\begin{tabular}{lrrrrrr}
\hline & min & Q1 & médiane & moyenne & Q3 & $\max$ \\
\hline CLC & 0,25 & 0,4 & 0,7 & 2,6 & 1,5 & 3857,0 \\
UCS (sol) & 1,8 & 13,6 & 39,9 & 199,8 & 170,9 & 4369,0 \\
NRA & 302,2 & 779,9 & 1138,0 & 1426,0 & 1964,0 & 4284,0 \\
communes & 0,3 & 6,0 & 10,4 & 15,1 & 18,2 & 169,7 \\
\hline
\end{tabular}

TABleau 10 - Répartition de la variable Corine Land Cover niveau 1

\begin{tabular}{llrr}
\hline code & libellé & effectif & fréquence (\%) \\
\hline 1 & territoires artificialisés & 652 & 2,6 \\
2 & territoires agricoles & 15385 & 60,8 \\
3 & forêts et milieux semi-naturels & 9148 & 36,2 \\
4 & zones humides & 4 & 0,0 \\
5 & surfaces en eau & 104 & 0,4 \\
\hline
\end{tabular}

TABleau 11 - Répartition de la variable Corine Land Cover niveau 2

\begin{tabular}{llrr}
\hline code & libellé & effectif & fréquence (\%) \\
\hline 11 & Zones urbanisées & 446 & 1,8 \\
12 & Zones industrielles ou commerciales & 122 & 0,5 \\
& et réseaux de communication & & \\
13 & Mines, décharges et chantiers & 44 & 0,2 \\
14 & Espaces verts artificialisés, non agricoles & 40 & 0,2 \\
21 & Terres arables & 6136 & 24,3 \\
22 & Cultures permanentes & 388 & 1,5 \\
23 & Prairies & 3101 & 12,3 \\
24 & Zones agricoles hétérogènes & 5760 & 22,8 \\
31 & Forêts & 6710 & 26,5 \\
32 & végétation arbustive et/ou herbacée & 1800 & 7,1 \\
33 & Espaces ouverts, sans ou avec peu de végétation & 638 & 2,5 \\
41 & Zones humides intérieures & 4 & 0,0 \\
51 & Eaux continentales & 104 & 0,4 \\
\hline
\end{tabular}

TABLEAU 12 - Répartition de la variable grandpole

\begin{tabular}{llrr}
\hline code & libellé & effectif & fréquence $(\%)$ \\
\hline 0 & non & 24066 & 95,1 \\
1 & oui & 1227 & 4,9 \\
\hline
\end{tabular}


TABleau 13 - Répartition de la variable texture du sol

\begin{tabular}{llrr}
\hline code & libellé & effectif & fréquence $(\%)$ \\
\hline 0 & Pas d'information & 248 & 1,0 \\
1 & Grossière $($ argile $<18 \%$ et sable $>65 \%)$ & 3653 & 14,4 \\
2 & Moyenne $(18 \%<$ argile $<35 \%$ et sable $>15 \%$, & 13642 & 53,9 \\
& ou argile $<18 \%$ et $15 \%<$ sable $<65 \%)$ & & \\
3 & Modérément fine $($ argile $<35 \%$ et sable $<15 \%)$ & 1843 & 7,3 \\
4 & Fine $(35 \%<$ argile $<60 \%)$ & 5907 & 23,4 \\
\hline
\end{tabular}

TABLEAU 14 - Répartition de la variable Matériau de base

\begin{tabular}{llrr}
\hline code & libellé & effectif & fréquence (\%) \\
\hline 0 & Pas d'information & 248 & 1,0 \\
1 & Dépôts alluviaux non-différenciés (ou dépôts glaciaires) & 5784 & 22,9 \\
2 & Roches calcaires & 6541 & 25,9 \\
3 & Matière argileuse & 724 & 2,9 \\
4 & Matière sableuse & 1031 & 4,1 \\
5 & Matière limoneuse & 177 & 0,7 \\
6 & Formations détritiques & 4071 & 16,1 \\
7 & Roches cristallines et migmatites & 6533 & 25,8 \\
8 & Roches volcaniques & 184 & 0,7 \\
\hline
\end{tabular}

TABLEAU 15 - Statistiques descriptives des variables explicatives quantitatives

\begin{tabular}{lrrrrrrr}
\hline & moyenne & écart-type & $\mathrm{Q} 1$ & médiane & $\mathrm{Q3}$ & $\min$ & $\max$ \\
\hline altitude $(\mathrm{m})$ & 492,4 & 467,1 & 198,1 & 317,3 & 608,3 & 59,2 & 3014,3 \\
température minimum $\left({ }^{\circ} \mathrm{C}\right)$ & $-9,7$ & 2,3 & $-10,8$ & $-9,4$ & $-7,8$ & $-15,6$ & $-6,6$ \\
température maximum $\left({ }^{\circ} \mathrm{C}\right)$ & 37,9 & 2,6 & 36,6 & 38,6 & 40,0 & 28,8 & 41,1 \\
température moyenne $\left({ }^{\circ} \mathrm{C}\right)$ & 11,5 & 1,8 & 10,7 & 12,1 & 12,7 & 4,7 & 13,4 \\
précipitations $(\mathrm{mm})$ & 759,2 & 160,6 & 611,5 & 715,6 & 793,9 & 537,1 & 1279,8 \\
vent moyen $(\mathrm{km} / \mathrm{h})$ & 10,2 & 2,8 & 8,2 & 9,6 & 12,4 & 3,3 & 16,9 \\
densité de pop. (hab./ $\left.\mathrm{km}^{2}\right)$ & 64,1 & 156,7 & 13,2 & 23,1 & 52,3 & 0,3 & 2807,2 \\
part de cadres (\%) & 4,8 & 4,2 & 2,0 & 4,1 & 6,7 & 0,0 & 33,3 \\
part d'agriculteurs (\%) & 5,8 & 6,1 & 1,2 & 4,0 & 8,5 & 0,0 & 66,7 \\
prix des terres (€ courant/ha) & 5273,5 & 1003,4 & 4510,0 & 5330,0 & 6270,0 & 3630,0 & 7310,0 \\
altitude des voisins (m) & 492,4 & 465,2 & 197,4 & 318,4 & 608,0 & 60,5 & 2866,3 \\
\hline
\end{tabular}

\subsection{Preuve de l'équation (5) page 7}

Montrons que :

$$
\mathrm{B}_{G}=\mathrm{B}_{J}+\frac{1}{2 n} \sum_{k \in I_{C}} \sum_{j \in I_{J}} \sum_{g \in I_{G_{j}}} \# G_{g}\left(\bar{z}_{g k}-\overline{\hat{p}}_{g k}-\bar{z}_{j k}+\overline{\hat{p}}_{j k}\right)^{2}
$$

Rappelons que :

$$
\mathrm{B}_{G}=\frac{1}{2 n} \sum_{k=1}^{K} \sum_{g \in I_{G}} \# G_{g}\left(\bar{z}_{g k}-\overline{\hat{p}}_{g k}\right)^{2}
$$

avec

$$
\begin{aligned}
& \bar{z}_{g k}=\frac{1}{\# G_{g}} \sum_{i \in G_{g}} z_{i k}=\text { fréquence observée de l'usage } k \text { dans le groupe } G_{g} \\
& \overline{\hat{p}}_{g k}=\frac{1}{\# G_{g}} \sum_{i \in G_{g}} \hat{p}_{i k}=\text { probabilité estimée de l'usage } k \text { dans le groupe } G_{g}
\end{aligned}
$$


les $I_{G_{j}}\left(j \in I_{J}\right)$ forment une partition de $I_{G}$ et $J_{j}=\cup_{g \in I_{G_{j}}} G_{g}$ avec $j \in I_{J}$, $\bar{z}_{j k}$ est la fréquence observée de l'usage $k$ dans le groupe $J_{j}$ :

$$
\bar{z}_{j k}=\frac{1}{\# J_{j}} \sum_{i \in J_{j}} z_{i k}=\frac{1}{\# J_{j}} \sum_{i \in \cup_{g \in I_{G_{j}}} G_{g}} z_{i k}=\frac{1}{\# J_{j}} \sum_{g \in I_{G_{j}}} \sum_{i \in G_{g}} z_{i k}=\frac{1}{\# J_{j}} \sum_{g \in I_{G_{j}}} \# G_{g} \bar{z}_{g k}
$$

$\overline{\hat{p}}_{j k}$ est la probabilité estimée de l'usage $k$ dans le groupe $J_{j}$ :

$$
\overline{\hat{p}}_{j k}=\frac{1}{\# J_{j}} \sum_{i \in J_{j}} \hat{p}_{i k}=\frac{1}{\# J_{j}} \sum_{g \in I_{G_{j}}} \# G_{g} \overline{\hat{p}}_{g k}
$$

Notons aussi que $\sum_{g \in I_{G_{j}}} \# G_{g}=\# J_{j}$.

Nous avons :

$$
\begin{aligned}
\mathrm{B}_{G}= & \frac{1}{2 n} \sum_{k \in I_{C}} \sum_{g \in G_{g}} \# G_{g}\left(\bar{z}_{g k}-\overline{\hat{p}}_{g k}\right)^{2} \\
= & \frac{1}{2 n} \sum_{k \in I_{C}} \sum_{j \in I_{J}} \sum_{g \in I_{G_{j}}} \# G_{g}\left(\bar{z}_{g k}-\overline{\hat{p}}_{g k}\right)^{2} \\
= & \frac{1}{2 n} \sum_{k \in I_{C}} \sum_{j \in I_{J}} \sum_{g \in I_{G_{j}}} \# G_{g}\left(\bar{z}_{g k}-\overline{\hat{p}}_{g k}-\bar{z}_{j k}+\overline{\hat{p}}_{j k}+\bar{z}_{j k}-\overline{\hat{p}}_{j k}\right)^{2} \\
= & \frac{1}{2 n} \sum_{k \in I_{C}} \sum_{j \in I_{J}} \sum_{g \in I_{G_{j}}} \# G_{g}\left(\bar{z}_{g k}-\overline{\hat{p}}_{g k}-\bar{z}_{j k}+\overline{\hat{p}}_{j k}\right)^{2}+\frac{1}{2 n} \sum_{k \in I_{C}} \sum_{j \in I_{J}} \sum_{g \in I_{G_{j}}} \# G_{g}\left(\bar{z}_{j k}-\overline{\hat{p}}_{j k}\right)^{2} \\
& +\frac{1}{n} \sum_{k \in I_{C}} \sum_{j \in I_{J}} \sum_{g \in I_{G_{j}}} \# G_{g}\left(\bar{z}_{g k}-\overline{\hat{p}}_{g k}-\bar{z}_{j k}+\overline{\hat{p}}_{j k}\right)\left(\bar{z}_{j k}-\overline{\hat{p}}_{j k}\right) \\
= & \frac{1}{2 n} \sum_{k \in I_{C}} \sum_{j \in I_{J}} \sum_{g \in I_{G_{j}}} \# G_{g}\left(\bar{z}_{g k}-\overline{\hat{p}}_{g k}-\bar{z}_{j k}+\overline{\hat{p}}_{j k}\right)^{2}+\frac{1}{2 n} \sum_{k \in I_{C}} \sum_{j \in I_{J}} \# J_{j}\left(\bar{z}_{j k}-\overline{\hat{p}}_{j k}\right)^{2} \\
+ & \frac{1}{n} \sum_{k \in I_{C}} \sum_{j \in I_{J}}\left(\bar{z}_{j k}-\overline{\hat{p}}_{j k}\right) \sum_{g \in I_{G_{j}}} \# G_{g}\left(\bar{z}_{g k}-\overline{\hat{p}}_{g k}-\frac{1}{\# J_{j}} \sum_{h \in I_{G j}} \# G_{h} \bar{z}_{h k}+\frac{1}{\# J_{j}} \sum_{h \in I_{G j}} \# G_{h} \overline{\hat{p}}_{h k}\right) \\
= & \frac{1}{2 n} \sum_{k \in I_{C}} \sum_{j \in I_{J}} \sum_{g \in I_{G_{j}}} \# G_{g}\left(\bar{z}_{g k}-\overline{\hat{p}}_{g k}-\bar{z}_{j k}+\overline{\hat{p}}_{j k}\right)^{2}+\frac{1}{2 n} \sum_{k \in I_{C}} \sum_{j \in I_{J}} \# J_{j}\left(\bar{z}_{j k}-\overline{\hat{p}}_{j k}\right)^{2} \\
+ & \frac{1}{n} \sum_{k \in I_{C}} \sum_{j \in I_{J}}\left(\bar{z}_{j k}-\overline{\hat{p}}_{j k}\right) \times\left[\sum _ { g \in I _ { G _ { j } } } \# \sum _ { j \in I _ { J } } \sum _ { g \in I _ { G _ { j } } } \# G _ { g } \left(\bar{z}_{g k}-\overline{\hat{p}}_{g k}-\bar{z}_{j k}+\sum_{g \in I_{G_{j}}} \# G_{g} \overline{\hat{p}}_{g k}-\sum_{h \in I_{G j}} \# \frac{1}{2 n} \sum_{k \in I_{C}} \sum_{j \in I_{J}} \# G_{j}\left(\bar{z}_{j k}-\overline{\hat{p}}_{h k}+\sum_{h \in I_{G j}} \# G_{h} \overline{\hat{p}}_{h k}\right]\right.\right. \\
\sum_{k} &
\end{aligned}
$$




\subsection{Tableaux des coefficients des MNL}

Niveau de significativité des coefficients : ${ }^{* * *}: 0,001^{* *}: 0,01 *: 0,05$.

TABleau 16 - Coefficients du modèle MNL-E

\begin{tabular}{lllll}
\hline variable & usage agricole & forêts & prairies & sols naturels \\
\hline constante & $-4,089$ & $-1,831$ & $-5,693^{* *}$ & $-2,808$ \\
CLC2-12 & $-1,267$ & $-1,144$ & $-1,788$ & 0,506 \\
CLC2-13 & 0,909 & 0,762 & 0,123 & $2,257^{* * *}$ \\
CLC2-14 & $-18,33$ & 0,142 & $-20,700$ & $-2,143$ \\
CLC2-21 & $4,359^{* * *}$ & $2,223^{* * *}$ & $3,271^{* * *}$ & $1,505^{* * *}$ \\
CLC2-22 & $3,882^{* * *}$ & $2,050^{* * *}$ & $2,479^{* * *}$ & $1,672^{* * *}$ \\
CLC2-23 & $2,871^{* * *}$ & $3,096^{* * *}$ & $4,124^{* * *}$ & $2,063^{* * *}$ \\
CLC2-24 & $3,155^{* * *}$ & $2,562^{* * *}$ & $3,362^{* * *}$ & $1,47^{* * *}$ \\
CLC2-31 & $2,300^{* * *}$ & $5,459^{* * *}$ & $2,827^{* * *}$ & $2,822^{* * *}$ \\
CLC2-32 & $1,989^{* * *}$ & $3,488^{* * *}$ & $3,605^{* * *}$ & $4,018^{* * *}$ \\
CLC2-33 & $-13,350$ & 1,820 & 1,292 & $3,912^{* * *}$ \\
CLC2-41 & 2,104 & 20,966 & 19,498 & 20,055 \\
CLC2-51 & $-15,465$ & $3,969^{* * *}$ & 1,968 & $5,175^{* * *}$ \\
altitude & 0,0003 & $0,002^{* * *}$ & $0,003^{* * *}$ & $0,002^{* * *}$ \\
prixterre & $0,0001^{* *}$ & $-0,0002^{* * *}$ & 0,0000 & $-0,0003^{* * *}$ \\
densite & $-0,0003$ & $-0,0004$ & $-0,001^{* *}$ & $-0,001^{*}$ \\
tempmini & $0,178^{* * *}$ & 0,064 & $-0,095^{*}$ & 0,041 \\
tempmaxi & $0,161^{* *}$ & $0,099^{*}$ & 0,071 & $0,162^{* *}$ \\
tempmoy & $-0,266^{*}$ & $-0,215^{*}$ & $-0,023$ & $-0,247^{*}$ \\
pluie & $-0,001$ & $-0,001^{*}$ & $-0,001$ & $-0,002^{* * *}$ \\
TEXT-1 & $-0,08$ & 0,497 & $-0,092$ & $-0,246$ \\
TEXT-2 & 0,129 & 0,183 & $-0,233$ & $-0,368$ \\
TEXT-3 & 0,413 & 0,406 & 0,100 & 0,178 \\
TEXT-4 & 0,285 & 0,204 & $-0,075$ & $-0,174$ \\
\hline & & & &
\end{tabular}

TABLEAU 17 - Coefficients du modèle MNL-Cm

\begin{tabular}{lllll}
\multicolumn{5}{c}{ TABLEAU $17-$ Coefficients du modèle MNL-Cm } \\
\hline variable & usage agricole & forêts & prairies & sols naturels \\
\hline constante & $-10,667^{* * *}$ & $-3,228$ & $-7,607^{* * *}$ & $-7,356^{* *}$ \\
CLC2-12 & $-1,261$ & $-1,173$ & $-1,814$ & 0,479 \\
CLC2-13 & 0,743 & 0,702 & 0,036 & $2,122^{* * *}$ \\
CLC2-14 & $-18,128$ & 0,304 & $-20,643$ & $-1,535$ \\
CLC2-21 & $4,461^{* * *}$ & $2,24^{* * *}$ & $3,295^{* * *}$ & $1,625^{* * *}$ \\
CLC2-22 & $3,989^{* * *}$ & $2,152^{* * *}$ & $2,46^{* * *}$ & $1,899^{* * *}$ \\
CLC2-23 & $2,938^{* * *}$ & $3,13^{* * *}$ & $4,125^{* * *}$ & $2,072^{* * *}$ \\
CLC2-24 & $3,209^{* * *}$ & $2,62^{* * *}$ & $3,368^{* * *}$ & $1,542^{* * *}$ \\
CLC2-31 & $2,357^{* * *}$ & $5,528^{* * *}$ & $2,821^{* * *}$ & $2,867^{* * *}$ \\
CLC2-32 & $2,067^{* * *}$ & $3,604^{* * *}$ & $3,634^{* * *}$ & $4,177^{* * *}$ \\
CLC2-33 & $-12,12$ & 1,884 & 1,474 & $4,269^{* * *}$ \\
CLC2-41 & 2,535 & 20,978 & 19,6 & 20,19 \\
CLC2-51 & $-15,422$ & $4,006^{* * *}$ & 2,001 & $5,262^{* * *}$ \\
altitude & $0,005^{*}$ & $0,004^{* *}$ & $0,006^{* * *}$ & $0,006^{* * *}$ \\
tempmini & $0,067^{*}$ & 0,045 & $-0,09^{* *}$ & 0,009 \\
ventmoy & $0,084^{* * *}$ & $-0,023$ & 0,006 & 0,001 \\
tempmaxi & $0,209^{* * *}$ & 0,028 & $0,094^{*}$ & $0,115^{*}$ \\
altitude_lag & $-0,005^{*}$ & $-0,002$ & $-0,004^{*}$ & $-0,003$ \\
\hline
\end{tabular}


TABlEau 18 - Coefficients du modèle MNL-S

\begin{tabular}{lllll}
\hline variable & usage agricole & forêts & prairies & sols naturels \\
\hline constante & $-1,814^{* * *}$ & $-2,688^{* * *}$ & $-3,035^{* * *}$ & $-2,813^{* * *}$ \\
CLC2-12 & $-1,259$ & $-1,123$ & $-1,782$ & 0,542 \\
CLC2-13 & 0,767 & 0,79 & 0,092 & $2,222^{* * *}$ \\
CLC2-14 & $-18,040$ & 0,495 & $-20,440$ & $-1,335$ \\
CLC2-21 & $4,513^{* * *}$ & $2,307^{* * *}$ & $3,323^{* * *}$ & $1,718^{* * *}$ \\
CLC2-22 & $3,967^{* * *}$ & $2,246^{* * *}$ & $2,495^{* * *}$ & $2,024^{* * *}$ \\
CLC2-23 & $2,848^{* * *}$ & $3,141^{* * *}$ & $4,221^{* * *}$ & $2,089^{* * *}$ \\
CLC2-24 & $3,165^{* * *}$ & $2,643^{* * *}$ & $3,464^{* * *}$ & $1,586^{* * *}$ \\
CLC2-31 & $2,271^{* * *}$ & $5,549^{* * *}$ & $2,892^{* * *}$ & $2,890^{* * *}$ \\
CLC2-32 & $1,945^{* * *}$ & $3,661^{* * *}$ & $3,681^{* * *}$ & $4,225^{* * *}$ \\
CLC2-33 & $-11,510$ & $2,235^{*}$ & 1,535 & $4,560^{* * *}$ \\
CLC2-41 & 2,612 & 21,006 & 19,870 & 20,264 \\
CLC2-51 & $-15,582$ & $3,989^{* * *}$ & $2,085^{*}$ & $5,252^{* * *}$ \\
altitude & $-0,001^{* * *}$ & $0,002^{* * *}$ & $0,003^{* * *}$ & $0,002^{* * *}$ \\
\hline
\end{tabular}

